\title{
STABLE OSCILLATING MOTIONS IN A MODEL OF A CHARGED-PARTICLE ACCELERATOR
}

\author{
L. D. PUSTYL'NIKOV \\ Dedicated to the memory of V. M. Alekseev
}

\begin{abstract}
This paper is devoted to a qualitative study of oscillating motions in Hamiltonian systems with five-dimensional phase space that depend periodically on the independent variable. It is proved that in the phase space there exists an open set of initial data giving rise to oscillating motions that are Lyapunov stable. The main example of such systems is the classical model of an accelerator of charged particles moving in a variable periodic electric field and a constant magnetic field, and the resulting oscillating motions lead to unbounded growth of the energy of the particles. It is established that the property of stability of the solutions is preserved under a small change in the Hamiltonian function.
\end{abstract}

\section{Contents}

Introduction

$\S 1$. Formulation and proof of Theorem 1

$\S 2$. The accelerator problem and formulation of the Basic Theorem

$\S 3$. Auxiliary lemmas

$\S 4$. Proof of the Basic Theorem

$\S 5$. Hamiltonian systems with smooth right-hand sides

$\S 6$. Preservation of stability of solutions under a small change of the Hamiltonian

$\S 7$. The limiting integrable system

\section{INTRODUCTION}

The main result in this paper is the construction of a substantial set of oscillating motions and a proof that these motions are stable in the five-dimensional extended phase space of nonlinear Hamiltonian systems with a Hamiltonian function that depends periodically on the independent variable.

A particular case of such systems describes the motion of a relativistic charged particle in a variable periodic electric field and a constant magnetic field orthogonal to it, and is a popular model of an accelerator ([1], [3, [2]). The basic physical and mathematical problems connected with this model involve finding initial conditions for which the energy of the particle tends to infinity. This problem arose and was studied more than 50 years ago on a physical level of rigor and in greater detail (in the dissertation of A. A. Kolomenskiü, Physics Institute of the Academy of Sciences of the USSR, 1950) in the very special case when the action of the electric field on the particle has the character of $\delta$-impulses on

1991 Mathematics Subject Classification. Primary 34D20, 78A35; Secondary 37Kxx, 34C15, 70H08, $70 \mathrm{H} 05,70 \mathrm{~F} 10$.

This work was carried out with the financial support of the Russian Foundation for Basic Research, grant no. 02-01-01067. 


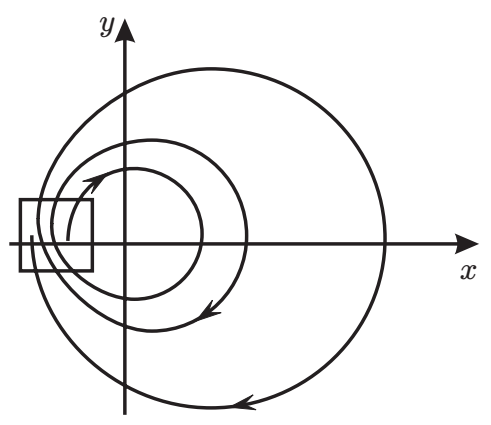

FigURE 1.

the line $y=0$ in the system (1), (2) in $\S 2$ (a resonator of infinitesimally small thickness). A rigorous solution of the problem was given in [4] in the case when the electric field depends only on the independent variable in an area of the configuration space having the shape of a rectangle $|x| \leq a,|y| \leq b(b>0)$ in the system (1), (2) (a resonator of finite thickness). The main result obtained in [4] says that for an open set of initial data in the five-dimensional phase space the energy of the particle tends to infinity, and the corresponding solutions describe oscillating motions (Figure 1).

A motion is said to be oscillating if in the configuration space it is not Lagrange stable (that is, the closure of the corresponding semitrajectory is not compact), but it does not tend to infinity. The concept of an oscillating motion came from celestial mechanics, and the possibility of such motions in the three-body problem was conjectured in 1922 by the French astronomer and mathematician Chazy [11. In 1959 Sitnikov proved the existence of oscillating motions in the three-body problem for the Kolmogorov model [12], in the cycle of papers 13. Alekseev proved the existence of both two-sided (that is, when $t \rightarrow \pm \infty, t$ being the time) and one-sided (that is, when $t \rightarrow+\infty$ ) oscillating motions in the Kolmogorov model, and in [8] the author obtained all these results in the $n$-body problem for $n \geq 3$. In [14] oscillating motions were found in a certain billiards problem. The sets of initial data in these papers giving rise to oscillating motions have Lebesgue measure zero in the three-dimensional phase space, and in [9] it was proved that the set of initial data giving rise to one-sided oscillating motions for the Kolmogorov model and its generalization in the problem of $n \geq 3$ bodies always has Lebesgue measure zero. At the same time, oscillating motions filling domains of positive (and even infinite) Lebesgue measure in three-dimensional and five-dimensional phase space have been observed in other dynamical systems with noncompact phase space that are similar to the indicated systems in celestial mechanics ([4], [5], [7]). The present article is a development of this direction. Here our attention is focused mainly on a problem arising naturally in the investigation of oscillating motions: the stability of solutions of Hamiltonian systems of general form that are nearly integrable. It is well known that the general case for such Hamiltonian systems with five-dimensional phase space is topological instability of solutions [15] in the sense that there exists a domain in a function space of such systems for which the solutions are not Lyapunov stable. In the present article we obtain a result opposite in meaning: there exists a domain in a function space of Hamiltonian systems with a natural norm for which the solutions are Lyapunov stable ( $(6)$. Just as in 15], the Hamiltonian function depends periodically on the independent variable, and the dimension of the extended phase space is equal to five. The closeness to integrable systems is manifested in the fact that there is a small parameter which takes the original system into an integrable system when it goes to zero $(\S 7)$. This work is a generalization of results in 4], where, however, the problem of stability was not considered explicitly. 
Our main results were reported at a session of the Moscow Mathematical Society in October 1994 and were announced in [10].

In $\S 1$ Roman numerals are used to number the formulas, but from $\S 2$ to the end of the paper the numbering of formulas is continuous and with Arabic numerals.

\section{$\S 1$. Formulation and Proof of Theorem 1}

Let us consider the Hamiltonian system

$$
\frac{d x}{d \varphi}=\frac{\partial H}{\partial Q}, \quad \frac{d y}{d \varphi}=\frac{\partial H}{\partial P}, \quad \frac{d Q}{d \varphi}=-\frac{\partial H}{\partial x}, \quad \frac{d P}{d \varphi}=-\frac{\partial H}{\partial y}
$$

in the extended phase space $x, y, Q, P, \varphi$ with the Hamiltonian function

$$
H=H(x, y, Q, P, \varphi)=\sqrt{1+\left(Q+\frac{\Omega y}{2}\right)^{2}+\left(P-\frac{\Omega x}{2}\right)^{2}}+F(x, y, \varphi),
$$

where $(x, Q)$ and $(y, P)$ are pairs of canonically conjugate dependent variables, $\varphi$ is an independent variable, and $\Omega>0$ is a constant. In this section we assume that the function $F(x, y, \varphi)$ satisfies the following conditions:

a)

b)

$$
\frac{\partial F}{\partial x}(x, y, \varphi) \equiv 0
$$

$$
\frac{\partial F}{\partial y}(x, y, \varphi)=\left\{\begin{array}{lll}
G(y, \varphi) & \text { if } & (x, y) \in \Pi, \\
0 & \text { if } & (x, y) \notin \Pi,
\end{array}\right.
$$

where $\Pi$ is a rectangle in the $(x, y)$-plane with the form

$$
\Pi=\{x, y:|x| \leq a,|y| \leq b\},
$$

$a>0, b>0$ being constants.

c) In the domain $\Pi$ the function $G(y, \varphi)$ is analytic with respect to the variables $y$ and $\varphi$ and has period $2 \pi$ with respect to $\varphi$.

Theorem 1. Suppose that the function $G(y, \varphi)$ satisfies the following conditions: there exist integers $k>0$ and $l_{0} \geq 4$ and a real number $\varphi_{0}$ with $0 \leq \varphi_{0}<2 \pi$ such that for $S(\tau)=-G\left(\tau-\varphi_{0}+b, \tau\right)$

1) $\int_{\varphi_{0}-2 b}^{\varphi_{0}} S(\tau) d \tau=\Omega k, \quad-\frac{\Omega}{\pi}<\frac{1}{2}\left(S\left(\varphi_{0}\right)-S\left(\varphi_{0}-2 b\right)\right) \stackrel{\text { def }}{=} \beta<0$

2) $\beta \neq-\frac{\Omega}{\pi}+\frac{\Omega}{2 \pi} \cos \left(\frac{2 \pi t}{\nu}\right)$, where $t=0, \pm 1, \ldots, \pm \nu, \quad \nu=1, \ldots, l_{0}$;

$$
\text { 3) } a_{0}(\beta)\left(\frac{d^{2} S}{d \tau^{2}}\left(\varphi_{0}\right)-\frac{d^{2} S}{d \tau^{2}}\left(\varphi_{0}-2 b\right)\right)+a_{1}(\beta)\left(\frac{d S}{d \tau}\left(\varphi_{0}\right)-\frac{d S}{d \tau}\left(\varphi_{0}-2 b\right)\right)^{2} \neq 0 \text {, }
$$

where $a_{0}(\beta)$ and $a_{1}(\beta)$ are the values at the point $s=\beta$ of some fixed functions $a_{0}(s)$ and $a_{1}(s)$ that are independent of the function $G(y, \varphi)$, and $a_{0}(\beta) \neq 0$. Then there exist a natural number $m_{0}$, a real number $\varkappa>0$, and a domain

$$
\begin{aligned}
\Gamma=\Gamma\left(\varkappa, m_{0}\right)= & \left\{\tilde{x}, \tilde{y}, \tilde{Q}, \tilde{P}, \tilde{\varphi}:|\tilde{x}| \leq \varkappa, \quad|\tilde{y}-b| \leq \varkappa,\left|\tilde{Q}+\frac{\Omega \tilde{y}}{2}-\varphi_{0} \Omega\right| \leq \varkappa,\right. \\
& \left.\left|\varphi-\varphi_{0}\right| \leq \varkappa, \quad\left|\sqrt{1+\left(\tilde{Q}+\frac{\Omega \tilde{y}}{2}\right)^{2}+\left(\tilde{P}-\frac{\Omega \tilde{x}}{2}\right)^{2}}-m_{0} \Omega\right| \leq \varkappa\right\}
\end{aligned}
$$


such that if $(\tilde{x}, \tilde{y}, \tilde{Q}, \tilde{P}, \tilde{\varphi}) \in \Gamma$ and $(x(\varphi), y(\varphi), Q(\varphi), P(\varphi))$ is a solution of the system (1) with initial data $x(\tilde{\varphi})=\tilde{x}, y(\tilde{\varphi})=\tilde{y}, Q(\tilde{\varphi})=\tilde{Q}, P(\tilde{\varphi})=\tilde{P}$, then the following assertions hold for $\varphi \geq \tilde{\varphi}$.

1. Each such solution intersects the domain $\Pi$ infinitely many times.

2. $\varlimsup_{\varphi \rightarrow \infty}|x(\varphi)|=\varlimsup_{\varphi \rightarrow \infty}|y(\varphi)|=\infty$.

3. $\lim _{\varphi \rightarrow \infty} \sqrt{1+\left(Q(\varphi)+\frac{\Omega y(\varphi)}{2}\right)^{2}+\left(P(\varphi)-\frac{\Omega x(\varphi)}{2}\right)^{2}}=\infty$.

4. For any $\varepsilon>0$ there exist numbers $\varkappa>0$ and $m^{*}>0$ such that if $m_{0}>m^{*}$, $(\tilde{x}, \tilde{y}, \tilde{Q}, \tilde{P}, \tilde{\varphi}) \in \Gamma\left(\varkappa, m_{0}\right)$, and $\left(\tilde{x}^{*}, \tilde{y}^{*}, \tilde{Q}^{*}, \tilde{P}^{*}, \tilde{\varphi}\right) \in \Gamma\left(\varkappa, m_{0}\right)$, then for the solutions $(x(\varphi), y(\varphi), Q(\varphi), P(\varphi))$ and $\left(x^{*}(\varphi), y^{*}(\varphi), Q^{*}(\varphi), P^{*}(\varphi)\right)$ of the system (I) with initial data $x(\tilde{\varphi})=\tilde{x}, y(\tilde{\varphi})=\tilde{y}, Q(\tilde{\varphi})=\tilde{Q}, P(\tilde{\varphi})=\tilde{P}$ and $x^{*}(\tilde{\varphi})=\tilde{x}^{*}$, $y^{*}(\tilde{\varphi})=\tilde{y}^{*}, Q^{*}(\tilde{\varphi})=\tilde{Q}^{*}, P^{*}(\tilde{\varphi})=\tilde{P}^{*}$ the assertions $1,2,3$ of Theorem 1 are satisfied, and for all $\varphi \geq \tilde{\varphi}$

$$
\left|x^{*}(\varphi)-x(\varphi)\right|+\left|y^{*}(\varphi)-y(\varphi)\right|+\left|Q^{*}(\varphi)-Q(\varphi)\right|+\left|P^{*}(\varphi)-P(\varphi)\right|<\varepsilon .
$$

Remark. The class of functions $G(y, \varphi)$ for which the conditions of Theorem 1 are valid contains an open set in the space of functions analytic with respect to $y$ and $\varphi$. It contains also the functions of the form $E(\varphi)=h \sin n \varphi$, where $n$ is an integer and $h$ belongs to an open subset of the line.

Proof of Theorem 1. We introduce the variables

$$
q=Q+\frac{\Omega y}{2}, \quad p=P-\frac{\Omega x}{2} .
$$

In the variables $x, y, q, p, \varphi$ the system of equations (I) takes the form of the system of equations (4) in $\S 2$, in which the function $D(x, y, \varphi)$ is $-\frac{\partial F}{\partial y}(x, y, \varphi)$ and has the form

$$
D(x, y, \varphi)=\left\{\begin{array}{lll}
E(y, \varphi) & \text { if } & (x, y) \in \Pi, \\
0 & \text { if } & (x, y) \notin \Pi,
\end{array}\right.
$$

where $E(y, \varphi)=-G(y, \varphi)$, and the functions $F(x, y, \varphi)$ and $G(y, \varphi)$ were introduced in (II) and (III). According to a condition of the Basic Theorem (§2), the function $S(\tau)$ satisfies the conditions 1)-3) of Theorem 1, and therefore it follows from the assertions 1-4 of the Basic Theorem and the equalities (IV) that all the assertions of Theorem 1 are valid, and hence Theorem 1 is proved.

\section{$\S 2$. The accelerator problem and formulation of the Basic Theorem}

We consider the system of four differential equations

$$
\begin{gathered}
\frac{d x}{d \varphi}=v, \quad \frac{d y}{d \varphi}=u, \\
\left\{\begin{array}{c}
\frac{d}{d \varphi}\left(\frac{u}{\sqrt{1-\left(u^{2}+v^{2}\right)}}\right)=-\Omega v+D(x, y, \varphi), \\
\frac{d}{d \varphi}\left(\frac{v}{\sqrt{1-\left(u^{2}+v^{2}\right)}}\right)=\Omega u,
\end{array}\right.
\end{gathered}
$$

where $\Omega>0$ is a given constant, and the function $D(x, y, \varphi)$ is given by $(\mathrm{V})$ and is thus nonzero only in the domain

$$
\Pi=\{x, y:|x| \leq a,|y| \leq b\}
$$

$(a>0, b>0)$, in which $D(x, y, \varphi)=E(y, \varphi)$, with $E(y, \varphi)$ a function analytic with respect to $y$ and $\varphi$ and having period $2 \pi$ with respect to $\varphi$. 
The system of equations (1), (2) describes the motion of a relativistic charged particle in the $(x, y)$-plane of three-dimensional Euclidean space, subject to the action of a constant magnetic field perpendicular to this plane and a variable electric field along the plane. Here $v, u$ are the components of the particle's velocity, measured in units of the speed of light, that is, the speed of light is equal to 1 and the modulus of the particle's velocity is $\sqrt{v^{2}+u^{2}}<1$. The magnetic field is characterized by the constant $\Omega$, and the electric field is nonzero only inside the rectangle $\Pi$ (a resonator of finite thickness) and is given by the analytic function $E(y, \varphi)$. The main result, obtained in the Basic Theorem stated below, is that for an open set of initial data in the five-dimensional extended phase space the energy $z=\frac{1}{\sqrt{1-u^{2}-v^{2}}}$ of the particle tends to infinity as $\varphi \rightarrow \infty$. The solutions of the system (1), (2) corresponding to these initial data give rise to the oscillating motions (Figure 1) defined in the Introduction and are Lyapunov stable. The particular case of the system (1), (2) in which the function $E$ introduced in (V) depends only on $\varphi$ was studied in [4].

We introduce the variables

$$
q=\frac{v}{\sqrt{1-u^{2}-v^{2}}}, \quad p=\frac{u}{\sqrt{1-u^{2}-v^{2}}}, \quad z=\frac{1}{\sqrt{1-u^{2}-v^{2}}}
$$

and express the system (1), (2) in the coordinates $x, y, q, p$ as follows:

$$
\left\{\begin{aligned}
\frac{d x}{d \varphi} & =\frac{q}{\sqrt{1+p^{2}+q^{2}}}, \\
\frac{d y}{d \varphi} & =\frac{p}{\sqrt{1+p^{2}+q^{2}}}, \\
\frac{d q}{d \varphi} & =\frac{\Omega p}{\sqrt{1+p^{2}+q^{2}}}, \\
\frac{d p}{d \varphi} & =-\frac{\Omega q}{\sqrt{1+p^{2}+q^{2}}}+D(x, y, \varphi) .
\end{aligned}\right.
$$

In the variables $q$ and $p$ the energy $z$ is given by $z=\sqrt{q^{2}+p^{2}+1}$.

Basic Theorem. Suppose that there exist integers $k>0$ and $l_{0} \geq 4$ and a real number $\varphi_{0}$ with $0 \leq \varphi_{0}<2 \pi$ such that for $S(\tau)=E\left(\tau-\varphi_{0}+b, \tau\right)$ the conditions 1$\left.\left.), 2\right), 3\right)$ of Theorem 1 hold.

Then there exist a natural number $m_{0}$, a real number $\varkappa>0$, and a domain

$$
\begin{aligned}
\Lambda=\Lambda\left(\varkappa, m_{0}\right) & \stackrel{\text { def }}{=}\left\{\tilde{x}, \tilde{y}, \tilde{q}, \tilde{p}, \tilde{\varphi}:|\tilde{x}| \leq \varkappa,|\tilde{y}-b| \leq \varkappa,\left|\tilde{q}-\varphi_{0} \Omega\right| \leq \varkappa,\right. \\
& \left.\left|\tilde{\varphi}-\varphi_{0}\right| \leq \varkappa,\left|\sqrt{1+\tilde{q}^{2}+\tilde{p}^{2}}-m_{0} \Omega\right| \leq \varkappa\right\}
\end{aligned}
$$

such that if $(\tilde{x}, \tilde{y}, \tilde{q}, \tilde{p}, \tilde{\varphi}) \in \Lambda$ and $(x(\varphi), y(\varphi), q(\varphi), p(\varphi))$ is a solution of the system (4) with initial data $x(\tilde{\varphi})=\tilde{x}, y(\tilde{\varphi})=\tilde{y}, q(\tilde{\varphi})=\tilde{q}, p(\tilde{\varphi})=\tilde{p}$, then the following assertions hold for $\varphi \geq \tilde{\varphi}$.

1. Each such solution intersects the domain $\Pi$ infinitely many times.

2. $\varlimsup_{\varphi \rightarrow \infty}|x(\varphi)|=\varlimsup_{\varphi \rightarrow \infty}|y(\varphi)|=\varlimsup_{\varphi \rightarrow \infty}|p(\varphi)|=\varlimsup_{\varphi \rightarrow \infty}|q(\varphi)|=\infty$.

3. $\lim _{\varphi \rightarrow \infty} \sqrt{1+q(\varphi)^{2}+p(\varphi)^{2}}=\infty$.

4. For any $\varepsilon>0$ there are numbers $\varkappa>0$ and $m^{*}>0$ such that if $m_{0}>m^{*}$, $(\tilde{x}, \tilde{y}, \tilde{q}, \tilde{p}, \tilde{\varphi}) \in \Lambda\left(\varkappa, m_{0}\right)$, and $\left(\tilde{x}^{*}, \tilde{y}^{*}, \tilde{q}^{*}, \tilde{p}^{*}, \tilde{\varphi}\right) \in \Lambda\left(\varkappa, m_{0}\right)$, then for the solutions $(x(\varphi), y(\varphi), q(\varphi), p(\varphi))$ and $\left(x^{*}(\varphi), y^{*}(\varphi), q^{*}(\varphi), p^{*}(\varphi)\right)$ of the system (4) with initial data $x(\tilde{\varphi})=\tilde{x}, y(\tilde{\varphi})=\tilde{y}, q(\tilde{\varphi})=\tilde{q}, p(\tilde{\varphi})=\tilde{p}$ and $x^{*}(\tilde{\varphi})=\tilde{x}^{*}, y^{*}(\tilde{\varphi})=\tilde{y}^{*}$, 
$q^{*}(\tilde{\varphi})=\tilde{q}^{*}, p^{*}(\tilde{\varphi})=\tilde{p}^{*}$ the assertions 1, 2, 3 of the Basic Theorem are valid, and for all $\varphi \geq \tilde{\varphi}$

$$
\left|x^{*}(\varphi)-x(\varphi)\right|+\left|y^{*}(\varphi)-y(\varphi)\right|+\left|q^{*}(\varphi)-q(\varphi)\right|+\left|p^{*}(\varphi)-p(\varphi)\right|<\varepsilon .
$$

The proof of the Basic Theorem is presented in $\S 4$. It uses auxiliary lemmas which are formulated and proved in $\S 3$.

\section{§3. Auxiliary lemmas}

Definition 1. Suppose that at the moment of time $\tilde{\varphi}$ the point $(\tilde{x}, \tilde{y})=(x(\tilde{\varphi}), y(\tilde{\varphi}))$ is in $\Pi, \tilde{y}=b$, its velocity at this time has the form $(\tilde{v}, \tilde{u})=(v(\tilde{\varphi}), u(\tilde{\varphi})), \tilde{u}>0$, and for $\varphi>\tilde{\varphi}$ the trajectory $(x(\varphi), y(\varphi), v(\varphi), u(\varphi))$ with initial data $(\tilde{x}, \tilde{y}, \tilde{v}, \tilde{u})$ leaves $\Pi$. Suppose also that there exist moments of time $\varphi^{\prime}>\tilde{\varphi}$ and $\tilde{\varphi}^{\prime}>\tilde{\varphi}$ closest to $\tilde{\varphi}$ with the properties that $\left(x\left(\varphi^{\prime}\right), y\left(\varphi^{\prime}\right)\right)=\left(x^{\prime}, y^{\prime}\right) \in \Pi$ and $\left(x\left(\tilde{\varphi}^{\prime}\right), y\left(\tilde{\varphi}^{\prime}\right)\right)=\left(\tilde{x}^{\prime}, \tilde{y}^{\prime}\right) \in \Pi, y^{\prime}=-b$ and $\tilde{y}^{\prime}=b$, and the velocities at the times $\varphi^{\prime}$ and $\tilde{\varphi}^{\prime}$ have the respective forms $\left(v^{\prime}, u^{\prime}\right)=\left(v\left(\varphi^{\prime}\right), u\left(\varphi^{\prime}\right)\right)$ and $\left(\tilde{v}^{\prime}, \tilde{u}^{\prime}\right)=\left(v\left(\tilde{\varphi}^{\prime}\right), u\left(\tilde{\varphi}^{\prime}\right)\right)$. Let

$$
\tilde{z}=\frac{1}{\sqrt{1-\left(\tilde{v}^{2}+\tilde{u}^{2}\right)}}, \quad z^{\prime}=\frac{1}{\sqrt{1-\left(u^{\prime}\right)^{2}-\left(v^{\prime}\right)^{2}}}, \quad \tilde{z}^{\prime}=\frac{1}{\sqrt{1-\left(\tilde{u}^{\prime}\right)^{2}-\left(\tilde{v}^{\prime}\right)^{2}}},
$$

and let mappings be defined by

$$
A:(\tilde{\varphi}, \tilde{z}) \longmapsto\left(\tilde{\varphi}^{\prime}, \tilde{z}^{\prime}\right), \quad A^{\prime}:(\tilde{\varphi}, \tilde{z}) \longmapsto\left(\varphi^{\prime}, z^{\prime}\right) .
$$

Lemma 1. Suppose that $\tilde{y}=b,-\varepsilon \leq \tilde{x} \leq \varepsilon$, and

$$
\bar{q} \geq \tilde{q}=\frac{\tilde{v}}{\sqrt{1-\tilde{u}^{2}-\tilde{v}^{2}}}>0, \quad \tilde{p}=\frac{\tilde{u}}{\sqrt{1-\tilde{u}^{2}-\tilde{v}^{2}}}, \quad \text { where } \quad 0<\varepsilon<a .
$$

Then there exists a constant $\bar{p}=\bar{p}(\varepsilon, \bar{q})$ independent of $\tilde{\varphi}, \tilde{q}$ (under the condition $\bar{q} \geq \tilde{q}$ ) such that if $\tilde{p} \geq \bar{p}$, then the points $\left(\varphi^{\prime}, z^{\prime}\right)=A^{\prime}(\tilde{\varphi}, \tilde{z})$ and $\left(\tilde{\varphi}^{\prime}, \tilde{z}^{\prime}\right)=A(\tilde{\varphi}, \tilde{z})$ are defined.

Proof. Let us study the behavior of solutions of the system consisting of the last two equations of the system (4) for the values $\varphi>\tilde{\varphi}$ for which $(x, y) \notin \Pi$ in the interval between $\tilde{\varphi}$ and $\varphi$. The solution of this system with initial data $(p(\tilde{\varphi}), q(\tilde{\varphi}))=(\tilde{p}, \tilde{q})$ has the following form for the indicated values of $\varphi$ :

$$
\left\{\begin{array}{l}
p(\varphi)=\sqrt{\tilde{z}^{2}-1} \cos \left(\frac{\Omega(\varphi-\tilde{\varphi}+\hat{\varphi})}{\tilde{z}}\right), \\
q(\varphi)=\sqrt{\tilde{z}^{2}-1} \sin \left(\frac{\Omega(\varphi-\tilde{\varphi}+\hat{\varphi})}{\tilde{z}}\right)
\end{array}\right.
$$

where

$$
\tilde{z}=\sqrt{\tilde{p}^{2}+\tilde{q}^{2}+1}, \quad \hat{\varphi}=\frac{\tilde{z}}{\Omega} \arcsin \frac{\tilde{q}}{\sqrt{\tilde{z}^{2}-1}} .
$$

Further, by (1),

$$
y(\varphi)=b+\int_{\tilde{\varphi}}^{\varphi} u(\varphi) d \varphi, \quad x(\varphi)=\tilde{x}+\int_{\tilde{\varphi}}^{\varphi} v(\varphi) d \varphi
$$

and in view of (3), (2)

$$
q(\varphi)=\tilde{q}+\Omega \int_{\tilde{\varphi}}^{\varphi} u(\varphi) d \varphi, \quad p(\varphi)=\tilde{p}-\Omega \int_{\tilde{\varphi}}^{\varphi} v(\varphi) d \varphi .
$$

Therefore, for $x(\varphi), y(\varphi)$ we have the expressions

$$
x(\varphi)=\tilde{x}-\frac{p(\varphi)-\tilde{p}}{\Omega}, \quad y(\varphi)=b+\frac{q(\varphi)-\tilde{q}}{\Omega},
$$


with the first equality in (7) valid only for those $\varphi>\tilde{\varphi}$ such that $(x, y) \notin \Pi$ in the interval between $\tilde{\varphi}$ and $\varphi$, and the second equality valid for any values $\varphi>\tilde{\varphi}$. If we now substitute the functions from (5) into (7) and use (6), then we get that

$$
\begin{aligned}
& x(\varphi)=\tilde{x}-\frac{\sqrt{\tilde{z}^{2}-1}}{\Omega}\left\{\cos \left(\frac{\Omega(\varphi-\tilde{\varphi}+\hat{\varphi})}{\tilde{z}}\right)-\cos \left(\frac{\Omega \hat{\varphi}}{\tilde{z}}\right)\right\}, \\
& y(\varphi)=b+\frac{\sqrt{\tilde{z}^{2}-1}}{\Omega}\left\{\sin \left(\frac{\Omega(\varphi-\tilde{\varphi}+\hat{\varphi})}{\tilde{z}}\right)-\sin \left(\frac{\Omega \hat{\varphi}}{\tilde{z}}\right)\right\} .
\end{aligned}
$$

By (6) there exists a $\bar{p}=\bar{p}(q)>0$ such that if $\tilde{p} \geq \bar{p}$, then

$$
0<\frac{\Omega \hat{\varphi}}{\tilde{z}}<\frac{\pi}{2} .
$$

Suppose that $\tilde{p} \geq \bar{p}$, and $\varphi^{\prime}>\tilde{\varphi}$ is the value closest to $\tilde{\varphi}$ such that

$$
\frac{3 \pi}{2}<\frac{\Omega\left(\varphi^{\prime}-\tilde{\varphi}+\hat{\varphi}\right)}{\tilde{z}}<2 \pi+\frac{\pi}{2}
$$

and the function $y(\varphi)$ in $(9)$ satisfies

$$
y\left(\varphi^{\prime}\right)=-b .
$$

Substituting (12) into (9), we have

$$
\sin \left(\frac{\Omega\left(\varphi^{\prime}-\tilde{\varphi}+\hat{\varphi}\right)}{\tilde{z}}\right)-\sin \left(\frac{\Omega \hat{\varphi}}{\tilde{z}}\right)=-\frac{2 b \Omega}{\sqrt{\tilde{z}^{2}-1}} .
$$

It follows from (11) and (6) that for large values of $\tilde{p}$ depending only on $\tilde{q}$ the quantity $\varphi^{\prime}$ satisfying (13) is determined from the equality

$$
\frac{\Omega\left(\varphi^{\prime}-\tilde{\varphi}+\hat{\varphi}\right)}{\tilde{z}}-2 \pi=\arcsin \left\{\sin \left(\frac{\Omega \hat{\varphi}}{\tilde{z}}\right)-\frac{2 b \Omega}{\sqrt{\tilde{z}^{2}-1}}\right\}
$$

which leads to the equality

$$
\varphi^{\prime}=\tilde{\varphi}-\hat{\varphi}+\frac{2 \pi \tilde{z}}{\Omega} \arcsin \left\{\sin \left(\frac{\Omega \hat{\varphi}}{\tilde{z}}\right)-\frac{2 b \Omega}{\sqrt{\tilde{z}^{2}-1}}\right\} .
$$

We consider the solution $(p(\varphi), q(\varphi))$ of the system of equations

$$
\frac{d p}{d \varphi}=-\frac{\Omega q}{\sqrt{1+p^{2}+q^{2}}}, \quad \frac{d q}{d \varphi}=\frac{\Omega p}{\sqrt{1+p^{2}+q^{2}}}
$$

with initial data $q(\tilde{\varphi})=\tilde{q}, p(\tilde{\varphi})=\tilde{p}$. This solution has the form (5). Let $\varphi^{*}$ be the value closest to $\tilde{\varphi}$ such that

$$
\varphi^{*}>\tilde{\varphi}, \quad q^{*} \stackrel{\text { def }}{=} q\left(\varphi^{*}\right)=-\tilde{q}, \quad p^{*} \stackrel{\text { def }}{=} p\left(\varphi^{*}\right)=\tilde{p} .
$$

We prove that $\varphi^{*}$ is determined from the equality

$$
\varphi^{*}=\tilde{\varphi}+\frac{2 \pi \tilde{z}}{\Omega}-2 \hat{\varphi} .
$$

Indeed, it follows from (5) that

$$
q^{*}=-\tilde{q}=q\left(\varphi^{*}\right)=\sqrt{\tilde{z}^{2}-1} \sin \left(\frac{\Omega\left(\varphi^{*}-\tilde{\varphi}+\hat{\varphi}\right)}{\tilde{z}}\right) .
$$

Therefore, since

$$
\tilde{q}=q(\tilde{\varphi})=\sqrt{\tilde{z}^{2}-1} \sin \left(\frac{\Omega \hat{\varphi}}{\tilde{z}}\right)
$$

we get the equality

$$
\frac{\Omega\left(\varphi^{*}-\tilde{\varphi}+\hat{\varphi}\right)}{\tilde{z}}=2 \pi-\frac{\Omega \hat{\varphi}}{\tilde{z}}
$$

which implies (15). 
For $q^{\prime}=q\left(\varphi^{\prime}\right)$ and $p^{\prime}=p\left(\varphi^{\prime}\right)$ we prove the equalities

$$
\begin{aligned}
\lim _{\tilde{p} \rightarrow \infty}\left(\varphi^{\prime}-\varphi^{*}\right) & =-2 b+2 \frac{\tilde{q}}{\Omega}, \\
\lim _{\tilde{p} \rightarrow \infty}\left(q^{\prime}-q^{*}\right) & =-2 b \Omega+2 \tilde{q}, \\
\lim _{\tilde{p} \rightarrow \infty}\left(p^{\prime}-p^{*}\right) & =0,
\end{aligned}
$$

in which the convergence is uniform with respect to $\tilde{\varphi}$ and $\tilde{q}$ with $0<\tilde{q} \leq \bar{q}$. Indeed, using (14), (15), and (6), we obtain (16), and since

$$
\begin{aligned}
& q^{\prime}-q^{*}=\sqrt{\tilde{z}^{2}-1}\left\{\sin \left(\frac{\Omega\left(\varphi^{\prime}-\tilde{\varphi}+\hat{\varphi}\right)}{\tilde{z}}\right)-\sin \left(\frac{\Omega\left(\varphi^{*}-\tilde{\varphi}+\hat{\varphi}\right)}{\tilde{z}}\right)\right\}, \\
& p^{\prime}-p^{*}=\sqrt{\tilde{z}^{2}-1}\left\{\cos \left(\frac{\Omega\left(\varphi^{\prime}-\tilde{\varphi}+\hat{\varphi}\right)}{\tilde{z}}\right)-\cos \left(\frac{\Omega\left(\varphi^{*}-\tilde{\varphi}+\hat{\varphi}\right)}{\tilde{z}}\right)\right\}
\end{aligned}
$$

in view of (5), (17) and (18) follow upon passing to the limit as $\tilde{z} \rightarrow \infty$ in the last two equations and using (14) and (16).

We now let $z^{\prime}=\tilde{z}$ and prove that for large $\bar{p}$

$$
A^{\prime}(\tilde{\varphi}, \tilde{z})=\left(\varphi^{\prime}, z^{\prime}\right)
$$

In view of (3) and (5)

$$
z^{2}(\varphi)=\frac{1}{1-u^{2}(\varphi)-v^{2}(\varphi)}=p^{2}(\varphi)+q^{2}(\varphi)+1=\tilde{z}^{2}
$$

for $\tilde{\varphi} \leq \varphi \leq \varphi^{\prime}$, and therefore it follows from Definition 1 and (12) that to prove (19) it suffices to prove the following two assertions:

1) if $\tilde{\varphi}<\varphi<\varphi^{\prime}$, then $(x(\varphi), y(\varphi)) \notin \Pi$,

2) $\left|x\left(\varphi^{\prime}\right)\right|<a$.

It follows from the definition of the number $\varphi^{\prime}$ and (9) that it suffices to prove 1) for those $\varphi$ such that

$$
\pi-\frac{\Omega \hat{\varphi}}{\tilde{z}} \leq \frac{\Omega(\varphi-\tilde{\varphi}+\hat{\varphi})}{\tilde{z}} \leq \pi+\alpha
$$

where

$$
\alpha=2 \pi-\frac{\Omega\left(\varphi^{\prime}-\tilde{\varphi}+\hat{\varphi}\right)}{\tilde{z}} .
$$

But in view of $(16),\left(15^{\prime}\right),(21)$, and (6) the quantity $\alpha$ can be made arbitrarily small by choosing $\bar{p}$ sufficiently large, so it follows from (8) that $x(\varphi)>a$ in the domain (20) for large $\bar{p}$, and this inequality proves the assertion 1).

To prove 2) we use (7) and (18). By (7), $x\left(\varphi^{\prime}\right)=\tilde{x}-\frac{p^{\prime}-\tilde{p}}{\Omega}$ and $-a<-\varepsilon \leq \tilde{x} \leq \varepsilon<a$ in view of a condition of Lemma 1 . Therefore, since $p^{*}=\tilde{p}$, we can use (18) to get that the assertion 2) holds for large $\bar{p}$. Thus, the equality (19) is proved.

Let us now consider the solution of the system

$$
\frac{d p}{d \varphi}=-\frac{\Omega q}{\sqrt{1+p^{2}+q^{2}}}+E(y(\varphi), \varphi), \quad \frac{d q}{d \varphi}=\frac{\Omega p}{\sqrt{1+p^{2}+q^{2}}}
$$

with initial data $p\left(\varphi^{\prime}\right)=p^{\prime}, q\left(\varphi^{\prime}\right)=q^{\prime}$ for $\varphi>\varphi^{\prime}$. We introduce the notation $\nu=\frac{1}{p^{\prime}}$, $\tau=p-p^{\prime}=p-\frac{1}{\nu}$ and substitute the expression $p=\tau+\frac{1}{\nu}$ for $p$ in (22). Then we get 
the system of equations

$$
\left\{\begin{aligned}
\frac{d \tau}{d \varphi} & =f(\tau, q, \varphi, \nu), \\
\frac{d q}{d \varphi} & =g(\tau, q, \nu)
\end{aligned}\right.
$$

depending on the parameter $\nu$, in which

$$
\begin{aligned}
& f=f(\tau, q, \varphi, \nu)=-\frac{\Omega q \nu}{\sqrt{\nu^{2}+(1+\tau \nu)^{2}+q^{2} \nu^{2}}}+E(y(\varphi, \nu), \varphi), \\
& g=g(\tau, q, \nu)=\Omega \frac{1+\tau \nu}{\sqrt{\nu^{2}+(1+\tau \nu)^{2}+q^{2} \nu^{2}}},
\end{aligned}
$$

and $x(\varphi)=x(\varphi, \nu)$ and $y(\varphi)=y(\varphi, \nu)$ are solutions of the equations

$$
\begin{aligned}
& \frac{d x}{d \varphi}=-\frac{q \nu}{\sqrt{\nu^{2}+(1+\tau \nu)^{2}+q^{2} \nu^{2}}}=f_{1}(\tau, q, \nu), \\
& \frac{d y}{d \varphi}=\frac{1+\tau \nu}{\sqrt{\nu^{2}+(1+\tau \nu)^{2}+q^{2} \nu^{2}}}=g_{1}(\tau, q, \nu)
\end{aligned}
$$

with initial data $x\left(\varphi^{\prime}\right)=x^{\prime}, y\left(\varphi^{\prime}\right)=y^{\prime}$. Furthermore, the initial data $p\left(\varphi^{\prime}\right)=p^{\prime}$, $q\left(\varphi^{\prime}\right)=q^{\prime}$ of the system (22) pass into the initial data $\tau\left(\varphi^{\prime}\right)=0, q\left(\varphi^{\prime}\right)=q^{\prime}$ of the system (23). By (24),

$$
\begin{aligned}
& f_{1}(\tau, q, 0)=\lim _{\nu \rightarrow 0} f_{1}(\tau, q, \nu)=0, \\
& g_{1}(\tau, q, 0)=\lim _{\nu \rightarrow 0} g_{1}(\tau, q, \nu)=1 .
\end{aligned}
$$

Therefore, the solution of the system (24) for $\nu=0$ and $\varphi>\varphi^{\prime}$ has the form $x(\varphi, 0)=x^{\prime}$, $y(\varphi, 0)=y^{\prime}+\varphi$. Using these expressions, we get that

$$
\begin{aligned}
f(\tau, q, \varphi, 0) & =\lim _{\nu \rightarrow 0} f(\tau, q, \varphi, \nu)=E\left(y^{\prime}+\varphi, \varphi\right), \\
g(\tau, q, 0) & =\lim _{\nu \rightarrow 0} g(\tau, q, \nu)=\Omega,
\end{aligned}
$$

and hence the solution $\tau(\varphi, \nu), q(\varphi, \nu)$ of the system (23) with $\nu=0$ and initial data $\tau\left(\varphi^{\prime}, 0\right)=0, q\left(\varphi^{\prime}, 0\right)=q^{\prime}$ has for $\varphi>\varphi^{\prime}$ the form

$$
\tau(\varphi, 0)=\int_{\varphi^{\prime}}^{\varphi} E\left(y^{\prime}+s, s\right) d s, \quad q(\varphi, 0)=q^{\prime}+\Omega\left(\varphi-\varphi^{\prime}\right)
$$

In view of $(25),(17),(18)$, the definition of $\nu$, and the continuously differentiable dependence of the solution of the system (23), (24) on the parameter and the initial data, there exists a $\bar{p}$ independent of $\tilde{q}$ and $\tilde{\varphi}$ such that for $\tilde{p} \geq \bar{p}$ and for some $\tilde{\varphi}^{\prime}>\varphi^{\prime}$ the solution $p(\varphi), q(\varphi)$ of the system (22) with initial data $p\left(\varphi^{\prime}\right)=p^{\prime}, q\left(\varphi^{\prime}\right)=q^{\prime}$ satisfies the conditions

$$
q\left(\tilde{\varphi}^{\prime}\right)=\tilde{q}, \quad \frac{d q}{d \varphi}(\varphi)>0, \quad \varphi^{\prime} \leq \varphi \leq \tilde{\varphi}^{\prime} ;
$$

let $\tilde{\varphi}^{\prime}$ be the smallest number with these properties, and let $\tilde{z}^{\prime}=z\left(\tilde{\varphi}^{\prime}\right)$. We prove that $\left(\tilde{\varphi}^{\prime}, \tilde{z}^{\prime}\right)=A(\tilde{\varphi}, \tilde{z})$ for large $\bar{p}$. To this end, it suffices in view of $(7),(26)$, and Definition 1 to prove that $(x(\varphi), y(\varphi)) \in \Pi$ for $\varphi^{\prime} \leq \varphi \leq \tilde{\varphi}^{\prime}$. We prove first of all that for $\varphi^{\prime} \leq \varphi \leq \tilde{\varphi}^{\prime}$

$$
|x(\varphi)|<a .
$$

Suppose that (27) does not hold. Consequently, there is a $\varphi=\bar{\varphi}$ closest to $\varphi^{\prime}$ with $\varphi^{\prime}<\bar{\varphi} \leq \tilde{\varphi}^{\prime}$ such that

$$
|x(\bar{\varphi})|=a .
$$


The inequality $|x(\varphi)| \leq a$ holds for $\varphi^{\prime} \leq \varphi \leq \bar{\varphi}$ and $\left(x^{\prime}, y^{\prime}\right) \in \Pi$, so it follows from (26), (7), and the definition of $\varphi^{\prime}$ that for $\varphi^{\prime} \leq \varphi \leq \bar{\varphi}$

$$
\frac{d y}{d \varphi}(\varphi)>0, \quad|y(\varphi)| \leq b, \quad(x(\varphi), y(\varphi)) \in \Pi
$$

Further, by (26), (29), (17), and the definition of the number $q^{*}$,

$$
0<\lim _{\tilde{p} \rightarrow \infty}\left(q(\bar{\varphi})-q^{\prime}\right) \leq \lim _{\tilde{p} \rightarrow \infty}\left(\tilde{q}-q^{\prime}\right)=\lim _{\tilde{p} \rightarrow \infty}\left\{\left(\tilde{q}-q^{*}\right)+\left(q^{*}-q^{\prime}\right)\right\}=2 b \Omega .
$$

Therefore, if $\bar{p}$ is sufficiently large, then from (25) and the continuously differentiable dependence of the solution of the systems (23), (24) on the parameter and the initial data we obtain the estimate

$$
\bar{\varphi}-\varphi^{\prime}<4 b .
$$

Hence, in view of (25) we have for large $\bar{p}$

$$
|\tau(\varphi, \nu)|<8 b \sup _{0 \leq t \leq 2 \pi}\left|E\left(y^{\prime}+t, t\right)\right|
$$

for $\varphi^{\prime} \leq \varphi \leq \bar{\varphi}$, and by (18) and the definition of $\tau$ we have for large $\bar{p}$

$$
|p(\varphi)|=\left|p^{\prime}+\tau(\varphi, \nu)\right|>\frac{\tilde{p}}{2}
$$

for $\varphi^{\prime} \leq \varphi \leq \bar{\varphi}$. We now express the variables $u$ and $v$ in (3) in terms of $q$ and $p$ :

$$
u=\frac{p}{\sqrt{1+p^{2}+q^{2}}}, \quad v=\frac{q}{\sqrt{1+p^{2}+q^{2}}} .
$$

Let $\tilde{\varepsilon}$ satisfy

$$
0<\tilde{\varepsilon}<a-\left|x\left(\varphi^{\prime}\right)\right|
$$

(in the proof of (19) it was shown that $\left|x\left(\varphi^{\prime}\right)\right|<a$, and for large $\bar{p}$ the difference $a-\left|x\left(\varphi^{\prime}\right)\right|$ is greater than some positive constant independent of $\bar{p}, \tilde{p}, \tilde{q}, \varphi)$. Then it follows from (31) and (1) that for large $\bar{p}$ the inequality $|v(\varphi)|<\frac{\varepsilon}{4 b}$ holds for $\varphi^{\prime} \leq \varphi \leq \bar{\varphi}$, and hence in view of (30) and (32)

$$
|x(\bar{\varphi})| \leq\left|x\left(\varphi^{\prime}\right)\right|+\left|x(\bar{\varphi})-x\left(\varphi^{\prime}\right)\right|=\left|x\left(\varphi^{\prime}\right)\right|+\left|\int_{\varphi^{\prime}}^{\bar{\varphi}} v(\varphi) d \varphi\right|<\left|x\left(\varphi^{\prime}\right)\right|+\tilde{\varepsilon}<a,
$$

which contradicts (28). Thus, the inequality (27) is proved.

Applying here the same arguments to $\tilde{\varphi}^{\prime}$ that were used above for the value $\bar{\varphi}$ defined in (28), we get that for large $\bar{p}$ we have (29) for $\varphi^{\prime} \leq \varphi \leq \tilde{\varphi}^{\prime}$, and this proves the equality $\left(\tilde{\varphi}^{\prime}, \tilde{z}^{\prime}\right)=A(\tilde{\varphi}, \tilde{z})$. Lemma 1 is proved.

In what follows we assume that $|\tilde{x}|<\bar{\varepsilon}<a, \tilde{q}$ satisfies $-\varkappa \leq \tilde{q}-l \leq \varkappa(l>0, \varkappa>0)$, $\tilde{p} \geq 2 \bar{p}$, and $\bar{\varepsilon}$ and $\bar{p}$ satisfy Lemma 1 with $\bar{q}=l+\varkappa$.

Definition 2. Let $\varphi_{0}=\frac{l}{\Omega}, \hat{E}(\varphi, s)=E(s-\varphi+b, s)$,

$$
\Delta(\varphi)=\int_{\varphi-2 b}^{\varphi} \hat{E}(\varphi, s) d s, \quad \Delta\left(\varphi_{0}\right)=\Omega k, \quad z_{0}=m \Omega, \quad z_{n}=z_{0}+n k \Omega,
$$

where $m>0$ and $k>0$ are integers, $n=0,1, \ldots$, with $m$ large enough that $\sqrt{z_{0}^{2}-(l+\varkappa)^{2}-1} \geq 2 \bar{p}$, and the numbers $\bar{q}$ and $\bar{p}$ were introduced in the statement of Lemma 1. 
Definition 3. For $n=1,2, \ldots$ we define transformations $\bar{A}_{n}, \bar{A}_{n}^{\prime}, \bar{A}_{n}^{*}, \bar{A}_{\infty, n}, \bar{A}_{\infty, n}^{\prime}$, $\bar{A}_{\infty, n}^{*}$ as follows:

$$
\begin{aligned}
& \bar{A}_{n}:(\tilde{\varphi}, \tilde{z}) \longmapsto\left(\tilde{\varphi}_{n}^{\prime}, \tilde{z}_{n}^{\prime}\right) \stackrel{\text { def }}{=} A\left(\tilde{\varphi}, \tilde{z}+z_{n-1}\right), \\
& \bar{A}_{n}^{\prime}:(\tilde{\varphi}, \tilde{z}) \longmapsto\left(\varphi_{n}^{\prime}, z_{n}^{\prime}\right) \stackrel{\text { def }}{=} A^{\prime}\left(\tilde{\varphi}, \tilde{z}+z_{n-1}\right), \\
& \bar{A}_{n}^{*}:(\tilde{\varphi}, \tilde{z}) \longmapsto\left(\varphi_{n}^{*}, z_{n}^{*}\right) \stackrel{\text { def }}{=}\left(\varphi_{n}^{*}, z_{n}^{\prime}\right), \\
& \bar{A}_{\infty, n}:(\tilde{\varphi}, \tilde{z}) \longmapsto\left(\tilde{\varphi}_{\infty, n}^{\prime}, \tilde{z}_{\infty, n}^{\prime}\right) \stackrel{\text { def }}{=}\left(\tilde{\varphi}+\frac{2 \pi\left(\tilde{z}+z_{n-1}\right)}{\Omega}, \tilde{z}+z_{n-1}+\Delta\left(\tilde{\varphi}_{\infty, n}^{\prime}\right)\right), \\
& \bar{A}_{\infty, n}^{\prime}:(\tilde{\varphi}, \tilde{z}) \longmapsto\left(\varphi_{\infty, n}^{\prime}, z_{\infty, n}^{\prime}\right) \stackrel{\text { def }}{=}\left(\tilde{\varphi}+\frac{2 \pi\left(\tilde{z}+z_{n-1}\right)}{\Omega}-2 b, z_{n}^{\prime}\right), \\
& \bar{A}_{\infty, n}^{*}:(\tilde{\varphi}, \tilde{z}) \longmapsto\left(\varphi_{\infty, n}^{*}, z_{\infty, n}^{*}\right) \stackrel{\text { def }}{=}\left(\tilde{\varphi}+\frac{2 \pi\left(\tilde{z}+z_{n-1}\right)}{\Omega}-2 \hat{\varphi}_{\infty}, z_{n}^{\prime}\right),
\end{aligned}
$$

where

$$
\begin{gathered}
\varphi_{n}^{*}=\tilde{\varphi}+\frac{2 \pi\left(\tilde{z}+z_{n-1}\right)}{\Omega}-2 \hat{\varphi}_{n-1}, \\
\hat{\varphi}_{n-1}=\frac{z+z_{n-1}}{\Omega} \arcsin \frac{\tilde{q}}{\sqrt{\left(\tilde{z}+z_{n-1}\right)^{2}-1}}, \\
\hat{\varphi}_{\infty}=\frac{\tilde{q}}{\Omega} .
\end{gathered}
$$

In Lemma 2 below the expression " $=O(f(n))$ " means " $\leq C|f(n)| "$, where $C$ is a constant independent of $n, \tilde{z}, \tilde{\varphi}, \tilde{q}$.

Lemma 2. There is an $\varepsilon_{0}>0$ such that for $n=1,2, \ldots$ the transformations $\bar{A}_{n}^{\prime}$ and $\bar{A}_{n}$ are defined in the domain

$$
\mathcal{U}_{\varepsilon_{0}}=\left\{\tilde{\varphi}, \tilde{z}:\left|\tilde{\varphi}-\varphi_{0}\right| \leq \varepsilon_{0},|\tilde{z}| \leq \varepsilon_{0}\right\}
$$

and

$$
\sup _{\substack{(\tilde{\varphi}, \tilde{z}) \in \mathcal{U}_{\varepsilon_{0}} \\-\varkappa \leq \tilde{q}-l \leq \varkappa}}\left|\bar{A}_{n}^{\prime}(\tilde{\varphi}, \tilde{z})-\bar{A}_{\infty, n}^{\prime}(\tilde{\varphi}, \tilde{z})\right|=O\left(n^{-2}\right),
$$

where $|(\varphi, z)|=\max \{|\varphi|,|z|\}$.

Proof. The fact that for small $\varepsilon_{0}>0$ the transformations $\bar{A}_{n}^{\prime}$ and $\bar{A}_{n}$ are defined in the domain $\mathcal{U}_{\varepsilon_{0}}$ follows from Definitions 2 and 3 and Lemma 1.

Further,

$$
\varphi_{n}^{\prime}-\varphi_{\infty, n}^{\prime}=\left(\varphi_{n}^{\prime}-\varphi_{n}^{*}\right)+\left(\varphi_{n}^{*}-\varphi_{\infty, n}^{*}\right)+\left(\varphi_{\infty, n}^{*}-\varphi_{\infty, n}^{\prime}\right) .
$$

By (14), (33), (34), and (6),

$$
\varphi_{n}^{\prime}-\varphi_{n}^{*}=\frac{\tilde{z}+z_{n-1}}{\Omega} \arcsin \left[\sin \frac{\Omega \hat{\varphi}_{n-1}}{\tilde{z}+z_{n-1}}-\frac{2 b \Omega}{\sqrt{\left(\tilde{z}+z_{n-1}\right)^{2}-1}}\right]+\hat{\varphi}_{n-1} .
$$

Therefore, according to (34)

$$
\varphi_{n}^{\prime}-\varphi_{n}^{*}=2 \hat{\varphi}_{n-1}-2 b+O\left(n^{-2}\right) .
$$

From the definitions of $\varphi_{n}^{*}, \varphi_{\infty, n}^{*}$, and $\varphi_{\infty, n}^{\prime}$ (see Definition 3 ),

$$
\varphi_{\infty, n}^{*}-\varphi_{\infty, n}^{\prime}=2 b-2 \hat{\varphi}_{\infty}, \quad \varphi_{n}^{*}-\varphi_{\infty, n}^{*}=2\left(\hat{\varphi}_{\infty}-\hat{\varphi}_{n-1}\right) .
$$

Combining the first of the equalities in (38) with (37), we get the estimate

$$
\varphi_{n}^{\prime}-\varphi_{n}^{*}+\varphi_{\infty, n}^{*}-\varphi_{\infty, n}^{\prime}=2\left(\hat{\varphi}_{n-1}-\hat{\varphi}_{\infty}\right)+O\left(n^{-2}\right)
$$


We now prove that

$$
\hat{\varphi}_{n-1}-\hat{\varphi}_{\infty}=O\left(n^{-2}\right) .
$$

In view of (34) and (35),

$$
\begin{aligned}
\left|\hat{\varphi}_{n-1}-\hat{\varphi}_{\infty}\right| & =\left|\frac{\tilde{z}+z_{n-1}}{\Omega} \arcsin \frac{\tilde{q}}{\sqrt{\left(\tilde{z}+z_{n-1}\right)^{2}-1}}-\hat{\varphi}_{\infty}\right| \\
& =\left|\frac{\tilde{z}+z_{n-1}}{\Omega}\left[\frac{\tilde{q}}{\sqrt{\left(\tilde{z}+z_{n-1}\right)^{2}-1}}+O\left(\frac{1}{\sqrt{\left(\left(\tilde{z}+z_{n-1}\right)^{2}-1\right)^{3 / 2}}}\right)\right]-\frac{\tilde{q}}{\Omega}\right| \\
& \leq \frac{\tilde{q}}{\Omega}\left|\frac{\tilde{z}+z_{n-1}}{\sqrt{\left(\tilde{z}+z_{n-1}\right)^{2}-1}}-1\right|+O\left(n^{-2}\right)=O\left(n^{-2}\right),
\end{aligned}
$$

which proves (40). Substituting (40) in (39) and combining the equality obtained with the second equality in (38), we get the assertion of Lemma 2 by virtue of (36).

Definition 4. For $n=0,1, \ldots$, we introduce the functions

$$
\begin{aligned}
F_{n}(\varphi, z)= & \frac{z+z_{n}+\tilde{z}}{\sqrt{\left(z+z_{n}+\tilde{z}\right)^{2}-1}} \cos \left(\frac{\Omega\left(\varphi+\varphi_{n+1}^{\prime}-\tilde{\varphi}+\hat{\varphi}_{n}-\frac{2 \pi\left(\tilde{z}+z_{n}\right)}{\Omega}\right)}{z+z_{n}+\tilde{z}}\right) \\
& +\sin \left(\frac{\Omega\left(\varphi+\varphi_{n+1}^{\prime}-\tilde{\varphi}+\hat{\varphi}_{n}-\frac{2 \pi\left(\tilde{z}+z_{n}\right)}{\Omega}\right)}{z+z_{n}+\tilde{z}}\right) \\
& \times \frac{\sqrt{\left(z+z_{n}+\tilde{z}\right)^{2}-1}}{\left(z+z_{n}+\tilde{z}\right)^{2}} \Omega\left(\varphi+\varphi_{n+1}^{\prime}-\tilde{\varphi}+\hat{\varphi}_{n}-\frac{2 \pi\left(\tilde{z}+z_{n}\right)}{\Omega}\right), \\
\Psi_{n}(\varphi)= & \frac{z^{(n)}(\varphi)+z_{n}+\tilde{z}}{\sqrt{\left(z^{(n)}(\varphi)+z_{n}+\tilde{z}\right)^{2}-1}} \frac{d z^{(n)}}{d \varphi} \\
& \times \sin \left(\frac{\Omega\left(\varphi+\varphi_{n+1}^{\prime}-\tilde{\varphi}+\hat{\varphi}_{n}-\frac{2 \pi\left(\tilde{z}+z_{n}\right)}{\Omega}\right)}{z^{(n)}(\varphi)+z_{n}+\tilde{z}}\right)-\frac{\sqrt{\left(z^{(n)}(\varphi)+z_{n}+\tilde{z}\right)^{2}-1}}{\left(z^{(n)}+z_{n}+\tilde{z}\right)^{2}} \\
& \times \frac{d z^{(n)}}{d \varphi} \Omega\left(\varphi+\varphi_{n+1}^{\prime}-\tilde{\varphi}+\hat{\varphi}_{n}-\frac{2 \pi\left(\tilde{z}+z_{n}\right)}{\Omega}\right) \\
& \times \cos \left(\frac{\Omega\left(\varphi+\varphi_{n+1}^{\prime}-\tilde{\varphi}+\hat{\varphi}_{n}-\frac{2 \pi\left(\tilde{z}+z_{n}\right)}{\Omega}\right)}{z^{(n)}(\varphi)+z_{n}+\tilde{z}}\right),
\end{aligned}
$$

where $z^{(n)}(\varphi)$ is the solution of the equation

$$
F_{n}\left(\varphi, z^{(n)}\right) \frac{d z^{(n)}}{d \varphi}=E\left(y\left(\varphi+\varphi_{n+1}^{\prime}\right), \varphi+\varphi_{n+1}^{\prime}\right)
$$

with initial data $z^{(n)}(0)=0$.

In what follows we assume that $(\tilde{\varphi}, \tilde{z}) \in \mathcal{U}_{\varepsilon_{0}}$. The expression $O\left(n^{\theta}\right)$ means that $\left|O\left(n^{\theta}\right)\right| \leq C n^{\theta}$, where $C$ is a constant independent of $n, \tilde{q}, \tilde{x}, \tilde{\varphi}, \tilde{z}, \varphi, z$; the letter $C$ with indices will stand for positive constants independent of $n, \tilde{q}, \tilde{x}, \tilde{\varphi}, \tilde{z}, \varphi, z$, and the number $m$ used in the definition of $z_{0}$ in Definition 2 will be assumed to be sufficiently large.

Lemma 3. Let $\hat{\mathcal{U}}_{\hat{\varepsilon}}=\{\varphi, z:|\varphi| \leq \hat{\varepsilon},|z| \leq \hat{\varepsilon}\}$, where $\hat{\varepsilon}>0$. Then

$$
\sup _{(\varphi, z) \in \hat{\mathcal{U}}_{\hat{\varepsilon}}}\left|F_{n}(\varphi, z)-1\right|=O\left(n^{-2}\right) .
$$


Proof. From the equality (41),

$$
F_{n}(\varphi, z)-1=\varkappa_{n}(\varphi, z)-1+\sigma_{n}(\varphi, z)
$$

where

$$
\begin{aligned}
\varkappa_{n}(\varphi, z)= & \frac{z+z_{n}+\tilde{z}}{\sqrt{\left(z+z_{n}+\tilde{z}\right)^{2}-1}} \cos \left(\frac{\Omega\left(\varphi+\varphi_{n+1}^{\prime}-\tilde{\varphi}+\hat{\varphi}_{n}-\frac{2 \pi\left(\tilde{z}+z_{n}\right)}{\Omega}\right)}{z+z_{n}+\tilde{z}}\right), \\
\sigma_{n}(\varphi, z)= & \frac{\sqrt{\left(z+z_{n}+\tilde{z}\right)^{2}-1}}{\left(z+z_{n}+\tilde{z}\right)^{2}} \Omega\left(\varphi+\varphi_{n+1}^{\prime}-\tilde{\varphi}+\hat{\varphi}_{n}-\frac{2 \pi\left(\tilde{z}+z_{n}\right)}{\Omega}\right) \\
& \times \sin \left(\frac{\Omega\left(\varphi+\varphi_{n+1}^{\prime}-\tilde{\varphi}+\hat{\varphi}_{n}-\frac{2 \pi\left(\tilde{z}+z_{n}\right)}{\Omega}\right)}{z+z_{n}+\tilde{z}}\right) .
\end{aligned}
$$

1) Estimate of $\left|\varkappa_{n}(\varphi, z)-1\right|$. From Definition $2, \varkappa_{n}(\varphi, z)-1$ is equal to

$$
\frac{((m+n k) \Omega+z+\tilde{z})^{2}\left(1+\beta_{n}(\varphi, z)\right)^{2}-((m+n k) \Omega+z+\tilde{z})^{2}-1}{\sqrt{((m+n k) \Omega+z+\tilde{z})^{2}-1} K} \stackrel{\text { def }}{=} \frac{L}{M},
$$

where $L$ and $M$ denote the numerator and denominator of the last fraction, and

$$
\begin{gathered}
\beta_{n}(\varphi, z)=\cos \left(\frac{\Omega\left(\varphi+\varphi_{n+1}^{\prime}-\tilde{\varphi}+\hat{\varphi}_{n}-\frac{2 \pi\left(\tilde{z}+z_{n}\right)}{\Omega}\right)}{z+z_{n}+\tilde{z}}\right)-1, \\
K=((m+n k) \Omega+z+\tilde{z})\left(1+\beta_{n}(\varphi, z)\right)+\sqrt{((m+n k) \Omega+z+\tilde{z})^{2}-1} .
\end{gathered}
$$

Let us estimate $L$ and $M$.

a) Estimate of $|L|$. We have

$$
|L| \leq\left|((m+n k) \Omega+z+\tilde{z})^{2}\left(2 \beta_{n}(\varphi, z)+\beta^{2}(\varphi, z)\right)+1\right|,
$$

and from Definition 3 and the equalities (15), (16) it follows that

$$
\left|\beta_{n}(\varphi, z)\right|=O\left(n^{-2}\right) .
$$

Therefore, $|L|=O(1)$.

b) Estimate of $|M|$. Using (44) and the definitions of $M$ and $K$, we get that

$$
|M| \geq C_{1} n k \Omega|K| \geq C_{2} n^{2} .
$$

Consequently, $\left|\varkappa_{n}(\varphi, z)-1\right|=O\left(n^{-2}\right)$.

2) Estimate of $\left|\sigma_{n}(\varphi, z)\right|$. By Definition 3 and (15), (16),

$$
\begin{array}{r}
\left|\sigma_{n}(\varphi, z)\right|=\mid \frac{\sqrt{((m+n k) \Omega+z+\tilde{z})^{2}-1}}{((m+n k) \Omega+z+\tilde{z})^{2}} \Omega\left(\varphi+\varphi_{n+1}^{\prime}-\tilde{\varphi}+\hat{\varphi}_{n}-\frac{2 \pi\left(\tilde{z}+z_{n}\right)}{\Omega}\right) \\
\times \sin \left(\frac{\Omega\left(\varphi+\varphi_{n+1}^{\prime}-\tilde{\varphi}+\hat{\varphi}_{n}-\frac{2 \pi\left(\tilde{z}+z_{n}\right)}{\Omega}\right)}{(m+n k) \Omega+z+\tilde{z}}\right) \mid=O\left(n^{-2}\right) .
\end{array}
$$

In view of the estimates 1) and 2) Lemma 3 is proved.

Lemma 4. Let $y(s)$ be the solution of the equation

$$
\frac{d y}{d s}=\frac{p(s)}{\sqrt{1+p^{2}(s)+q^{2}(s)}}
$$


with initial data $y\left(\varphi_{n+1}^{\prime}\right)=-b$, and let $z_{\infty}^{(n)}$ be the solution of the equation

$$
\frac{d z_{\infty}^{(n)}}{d \varphi}=E\left(y\left(\varphi+\varphi_{\infty, n+1}^{\prime}\right), \varphi+\varphi_{\infty, n+1}^{\prime}\right)
$$

with initial data $z_{\infty}^{(n)}(0)=0$. Then

$$
\sup _{0 \leq \varphi \leq \hat{\varepsilon}}\left|z^{(n)}(\varphi)-z_{\infty}^{(n)}(\varphi)\right|=O\left(n^{-2}\right) .
$$

(Here the quantity $\varphi_{\infty, n+1}^{\prime}$ is taken from Definition 3 , and $z^{(n)}(\varphi)$ is the solution of the equation (43).)

Proof. Let us rewrite the equation (43) in the form

$$
\frac{d z^{(n)}}{d \varphi}=E_{n}\left(\varphi, z^{(n)}\right), \quad \text { where } \quad E_{n}\left(\varphi, z^{(n)}\right)=\frac{E\left(y\left(\varphi+\varphi_{n+1}^{\prime}\right), \varphi+\varphi_{n+1}^{\prime}\right)}{F_{n}\left(\varphi, z^{(n)}\right)} .
$$

Further, we have

$$
\left|z^{(n)}(\hat{\varphi})-z_{\infty}^{(n)}(\hat{\varphi})\right| \leq \int_{0}^{\hat{\varphi}}\left|E\left(y\left(\varphi+\varphi_{\infty, n+1}^{\prime}\right), \varphi+\varphi_{\infty, n+1}^{\prime}\right)-E_{n}\left(\varphi, z^{(n)}(\varphi)\right)\right| d \varphi .
$$

By Lemmas 2 and 3,

$$
\begin{aligned}
& \left|E_{n}\left(\varphi, z^{(n)}(\varphi)\right)-E\left(y\left(\varphi+\varphi_{\infty, n+1}^{\prime}\right), \varphi+\varphi_{\infty, n+1}^{\prime}\right)\right| \\
& \leq\left|E\left(y\left(\varphi+\varphi_{n+1}^{\prime}\right), \varphi+\varphi_{n+1}^{\prime}\right)\right| \cdot\left|\frac{1}{F_{n}\left(\varphi, z^{(n)}(\varphi)\right)}-1\right| \\
& \quad+\left|E\left(y\left(\varphi+\varphi_{n+1}^{\prime}\right), \varphi+\varphi_{n+1}^{\prime}\right)-E\left(y\left(\varphi+\varphi_{\infty, n+1}^{\prime}\right), \varphi+\varphi_{\infty, n+1}^{\prime}\right)\right|=O\left(n^{-2}\right),
\end{aligned}
$$

and thus Lemma 4 follows from (46).

\section{Lemma 5 .}

$$
\sup _{0 \leq \varphi \leq \hat{\varepsilon}}\left|\Psi_{n}(\varphi)\right|=O\left(n^{-2}\right) .
$$

Proof. According to Definition 4,

$$
\begin{aligned}
\Psi_{n}(\varphi)= & \frac{d z^{(n)}}{d \varphi} \cos \left(\frac{\Omega\left(\varphi+\varphi_{n+1}^{\prime}-\tilde{\varphi}+\hat{\varphi}_{n}-\frac{2 \pi\left(\tilde{z}+z_{n}\right)}{\Omega}\right)}{z^{(n)}(\varphi)+z_{n}+\tilde{z}}\right) \\
& \times\left\{\frac{z^{(n)}(\varphi)+z_{n}+\tilde{z}}{\sqrt{\left(z^{(n)}(\varphi)+z_{n}+\tilde{z}\right)^{2}-1}}\left(\frac{\Omega\left(\varphi+\varphi_{n+1}^{\prime}-\tilde{\varphi}+\hat{\varphi}_{n}-\frac{2 \pi\left(\tilde{z}+z_{n}\right)}{\Omega}\right)}{z^{(n)}(\varphi)+z_{n}+\tilde{z}}+\gamma_{n}(\varphi)\right)\right. \\
& \left.-\frac{\sqrt{\left(z^{(n)}(\varphi)+z_{n}+\tilde{z}\right)^{2}-1}}{\left(z^{(n)}(\varphi)+z_{n}+\tilde{z}\right)^{2}} \Omega\left(\varphi+\varphi_{n+1}^{\prime}-\tilde{\varphi}+\hat{\varphi}_{n}-\frac{2 \pi\left(\tilde{z}+z_{n}\right)}{\Omega}\right)\right\},
\end{aligned}
$$

where

$$
\begin{aligned}
\gamma_{n}(\varphi)= & \tan \left(\frac{\Omega\left(\varphi+\varphi_{n+1}^{\prime}-\tilde{\varphi}+\hat{\varphi}_{n}-\frac{2 \pi\left(\tilde{z}+z_{n}\right)}{\Omega}\right)}{z^{(n)}(\varphi)+z_{n}+\tilde{z}}\right) \\
& -\frac{\Omega\left(\varphi+\varphi_{n+1}^{\prime}-\tilde{\varphi}+\hat{\varphi}_{n}-\frac{2 \pi\left(\tilde{z}+z_{n}\right)}{\Omega}\right)}{z^{(n)}(\varphi)+z_{n}+\tilde{z}} .
\end{aligned}
$$


Therefore, using Lemma 4, we have

$$
\begin{aligned}
\left|\Psi_{n}(\varphi)\right| & <C_{3}\left\{\left|\frac{1}{\sqrt{\left(z^{(n)}(\varphi)+z_{n}+\tilde{z}\right)^{2}-1}}-\frac{\sqrt{\left(z^{(n)}(\varphi)+z_{n}+\tilde{z}\right)^{2}-1}}{\left(z^{(n)}(\varphi)+z_{n}+\tilde{z}\right)^{2}}\right|+\left|\gamma_{n}(\varphi)\right|\right\} \\
& <C_{4}\left\{\frac{1}{\left[\left((m+n k) \Omega+z^{(n)}(\varphi)+\tilde{z}\right)^{2}-1\right]^{5 / 2}}+\left|\gamma_{n}(\varphi)\right|\right\} .
\end{aligned}
$$

Since $\left|\gamma_{n}(\varphi)\right|=O\left(n^{-2}\right)$, Lemma 5 follows from the last inequality.

Lemma 6. Let $\left(\hat{p}^{(n)}(\varphi), \hat{q}^{(n)}(\varphi)\right)$ be the solution of the system

$$
\begin{aligned}
& \frac{d \hat{p}^{(n)}}{d \varphi}=-\frac{\Omega\left(\hat{q}^{(n)}+q_{n}^{\prime}\right)}{\sqrt{1+\left(\hat{p}^{(n)}+p_{n}^{\prime}\right)^{2}+\left(\hat{q}^{(n)}+q_{n}^{\prime}\right)^{2}}}+E\left(y\left(\varphi+\varphi_{n+1}^{\prime}\right), \varphi+\varphi_{n+1}^{\prime}\right), \\
& \frac{d \hat{q}^{(n)}}{d \varphi}=\frac{\Omega\left(\hat{p}^{(n)}+p_{n}^{\prime}\right)}{\sqrt{1+\left(\hat{p}^{(n)}+p_{n}^{\prime}\right)^{2}+\left(\hat{q}^{(n)}+q_{n}^{\prime}\right)^{2}}}+\Psi_{n}(\varphi)
\end{aligned}
$$

with initial data $\hat{p}^{(n)}(0)=0, \hat{q}^{(n)}(0)=0$, and let $\left(p^{(n)}(\varphi), q^{(n)}(\varphi)\right)$ be the solution of the system

$$
\begin{aligned}
\frac{d p^{(n)}}{d \varphi} & =-\frac{\Omega\left(q^{(n)}+q_{n}^{\prime}\right)}{\sqrt{1+\left(p^{(n)}+p_{n}^{\prime}\right)^{2}+\left(q^{(n)}+q_{n}^{\prime}\right)^{2}}}+E\left(y\left(\varphi+\varphi_{n+1}^{\prime}\right), \varphi+\varphi_{n+1}^{\prime}\right), \\
\frac{d q^{(n)}}{d \varphi} & =\frac{\Omega\left(p^{(n)}+p_{n}^{\prime}\right)}{\sqrt{1+\left(p^{(n)}+p_{n}^{\prime}\right)^{2}+\left(q^{(n)}+q_{n}^{\prime}\right)^{2}}}
\end{aligned}
$$

with initial data $p^{(n)}(0)=0, q^{(n)}(0)=0$, where

$$
\begin{aligned}
& p_{n}^{\prime}=\sqrt{\left(z_{n}+\tilde{z}\right)^{2}-1} \cos \left(\frac{\Omega\left(\varphi_{n+1}^{\prime}-\tilde{\varphi}+\hat{\varphi}_{n}\right)}{z_{n}+\tilde{z}}\right), \\
& q_{n}^{\prime}=\sqrt{\left(z_{n}+\tilde{z}\right)^{2}-1} \sin \left(\frac{\Omega\left(\varphi_{n+1}^{\prime}-\tilde{\varphi}+\hat{\varphi}_{n}\right)}{z_{n}+\tilde{z}}\right),
\end{aligned}
$$

and the function $\Psi_{n}(\varphi)$ was introduced in Definition 4 . Next, let $\left(\tilde{\varphi}_{n+1}^{\prime}, \tilde{z}_{n+1}^{\prime}\right)=\bar{A}_{n}(\tilde{\varphi}, \tilde{z})$ (see Definition 3). Then the following statements hold:

1) a point $\left(\hat{\varphi}_{n+1}^{\prime}, \hat{z}_{n+1}^{\prime}\right)$ is defined such that $\hat{\varphi}_{n+1}^{\prime}>0$,

$$
\hat{q}^{(n)}\left(\hat{\varphi}_{n+1}^{\prime}\right)+q_{n}^{\prime}=\tilde{q}, \quad \hat{z}_{n+1}^{\prime}=\sqrt{\tilde{q}^{2}+\left(\hat{p}^{(n)}\left(\hat{\varphi}_{n+1}^{\prime}\right)+p_{n}^{\prime}\right)^{2}+1},
$$

2) and $\hat{\varphi}_{n+1}^{\prime}$ is the number closest to 0 with these properties;

$$
\left|\hat{\varphi}_{n+1}^{\prime}-\left(\tilde{\varphi}_{n+1}^{\prime}-\varphi_{n+1}^{\prime}\right)\right|+\left|\hat{z}_{n+1}^{\prime}-\tilde{z}_{n+1}^{\prime}\right|=O\left(n^{-2}\right) ;
$$

3) for $0 \leq \varphi \leq \tilde{\varphi}_{n+1}^{\prime}-\varphi_{n+1}^{\prime}+\hat{\delta}$

$$
\sup _{0 \leq \varphi \leq \tilde{\varphi}_{n+1}^{\prime}-\varphi_{n+1}^{\prime}+\hat{\delta}}\left(\left|\hat{q}^{(n)}(\varphi)-q^{(n)}(\varphi)\right|+\left|\hat{p}^{(n)}(\varphi)-p^{(n)}(\varphi)\right|\right)=O\left(n^{-2}\right),
$$

where $\hat{\delta}>0$ is a constant independent of $n, \tilde{q}, \tilde{x}, \tilde{\varphi}, \tilde{z}$.

Proof. We rewrite the systems (47), (48) in the form

$$
\begin{aligned}
\frac{d \hat{p}^{(n)}}{d \varphi} & =\hat{f}_{1}\left(\hat{p}^{(n)}, \hat{q}^{(n)}, \varphi\right)=\hat{f}_{1}, & & \frac{d \hat{q}^{(n)}}{d \varphi}=\hat{f}_{2}\left(\hat{p}^{(n)}, \hat{q}^{(n)}, \varphi\right)=\hat{f}_{2}, \\
\frac{d p^{(n)}}{d \varphi} & =f_{1}\left(p^{(n)}, q^{(n)}, \varphi\right)=f_{1}, & & \frac{d q^{(n)}}{d \varphi}=f_{2}\left(p^{(n)}, q^{(n)}, \varphi\right)=f_{2} .
\end{aligned}
$$


Assertion 1 follows from Lemma 5 and Definition 3, and the solution of the system (47) is defined for $0 \leq \varphi \leq \tilde{\varphi}_{n+1}^{\prime}-\varphi_{n+1}^{\prime}+\hat{\delta}$, where $\hat{\delta}$ is a positive constant independent of $n, \tilde{q}, \tilde{x}, \tilde{\varphi}, \tilde{z}$. Further, we have

$$
\hat{q}^{(n)}\left(\hat{\varphi}_{n+1}^{\prime}\right)=\int_{0}^{\hat{\varphi}_{n+1}^{\prime}} \hat{f}_{2}\left(\hat{p}^{(n)}(\varphi), \hat{q}^{(n)}(\varphi), \varphi\right) d \varphi=\tilde{q}-q_{n}^{\prime},
$$

and in view of (7), (48), and (50)

$$
q^{(n)}\left(\tilde{\varphi}_{n+1}^{\prime}-\varphi_{n+1}^{\prime}\right)=\int_{0}^{\tilde{\varphi}_{n+1}^{\prime}-\varphi_{n+1}^{\prime}} f_{2}\left(p^{(n)}(\varphi), q^{(n)}(\varphi), \varphi\right) d \varphi=\tilde{q}-q_{n}^{\prime} .
$$

This gives us that

$$
\begin{aligned}
\int_{0}^{\tilde{\varphi}_{n+1}^{\prime}-\varphi_{n+1}^{\prime}} & \left\{f_{2}\left(p^{(n)}(\varphi), q^{(n)}(\varphi), \varphi\right)-\hat{f}_{2}\left(\hat{p}^{(n)}(\varphi), \hat{q}^{(n)}(\varphi), \varphi\right)\right\} d \varphi \\
= & \int_{\tilde{\varphi}_{n+1}^{\prime}-\varphi_{n+1}^{\prime}}^{\hat{\varphi}_{n+1}^{\prime}} \hat{f}_{2}\left(\hat{p}^{(n)}(\varphi), \hat{q}^{(n)}(\varphi), \varphi\right) d \varphi .
\end{aligned}
$$

Let

$$
\begin{aligned}
& J^{(0)} \stackrel{\text { def }}{=} \sup _{0 \leq \tau \leq \tilde{\varphi}_{n+1}^{\prime}-\varphi_{n+1}^{\prime}+\hat{\delta}}\left|\int_{0}^{\tau}\left\{f_{2}\left(p^{(n)}(\varphi), q^{(n)}(\varphi), \varphi\right)-\hat{f}_{2}\left(\hat{p}^{(n)}(\varphi), \hat{q}^{(n)}(\varphi), \varphi\right)\right\} d \varphi\right| \\
&+\sup _{0 \leq \tau \leq \hat{\varphi}_{n+1}^{\prime}-\varphi_{n+1}^{\prime}+\hat{\delta}}\left|\int_{0}^{\tau}\left\{f_{1}\left(p^{(n)}(\varphi), q^{(n)}(\varphi), \varphi\right)-\hat{f}_{1}\left(\hat{p}^{(n)}(\varphi), \hat{q}^{(n)}(\varphi), \varphi\right)\right\} d \varphi\right| \\
&= \sup _{0 \leq \varphi \leq \tilde{\varphi}_{n+1}^{\prime}-\varphi_{n+1}^{\prime}+\hat{\delta}}\left|\hat{q}^{(n)}(\varphi)-q^{(n)}(\varphi)\right|+\sup _{0 \leq \varphi \leq \tilde{\varphi}_{n+1}^{\prime}-\varphi_{n+1}^{\prime}+\hat{\delta}}\left|\hat{p}^{(n)}(\varphi)-p^{(n)}(\varphi)\right| .
\end{aligned}
$$

Then

$$
\begin{aligned}
J^{(0) \leq} \sup _{0 \leq \tau \leq \tilde{\varphi}_{n+1}^{\prime}-\varphi_{n+1}^{\prime}+\hat{\delta}} \mid & \left.f_{2}\right|_{1}\left(\sup _{0 \leq \varphi \leq \tau}\left|p^{(n)}(\varphi)-\hat{p}^{(n)}(\varphi)\right|\right. \\
& \left.+\sup _{0 \leq \varphi \leq \tau}\left|q^{(n)}(\varphi)-\hat{q}^{(n)}(\varphi)\right|\right)\left(\tilde{\varphi}_{n+1}^{\prime}-\varphi_{n+1}^{\prime}+\hat{\delta}\right) \\
+ & \sup _{0 \leq \tau \leq \tilde{\varphi}_{n+1}^{\prime}-\varphi_{n+1}^{\prime}+\hat{\delta}}\left|f_{1}\right|_{1}\left(\sup _{0 \leq \varphi \leq \tau}\left|p^{(n)}(\varphi)-\hat{p}^{(n)}(\varphi)\right|\right. \\
& \left.+\sup _{0 \leq \varphi \leq \tau}\left|q^{(n)}(\varphi)-\hat{q}^{(n)}(\varphi)\right|\right)\left(\tilde{\varphi}_{n+1}^{\prime}-\varphi_{n+1}^{\prime}+\hat{\delta}\right) \\
+ & \sup _{0 \leq \tau \leq \tilde{\varphi}_{n+1}^{\prime}-\varphi_{n+1}^{\prime}+\hat{\delta}}\left|\int_{0}^{\tau} \Psi_{n}(\varphi) d \varphi\right|,
\end{aligned}
$$

where

$$
\begin{aligned}
\left|f_{i}\right|_{1}= & \sup _{\substack{0 \leq \varphi_{1}, \varphi_{2} \leq \tilde{\varphi}_{n+1}^{\prime}-\varphi_{n+1}^{\prime}+\hat{\delta}}} \max \left\{\left|\frac{\partial f_{i}}{\partial p_{n}}\left(p^{(n)}\left(\varphi_{1}\right), q^{(n)}\left(\varphi_{1}\right), \varphi_{1}\right)\right|,\right. \\
& \left.\left|\frac{\partial f_{i}}{\partial q_{n}}\left(p^{(n)}\left(\varphi_{2}\right), q^{(n)}\left(\varphi_{2}\right), \varphi_{2}\right)\right|\right\}
\end{aligned}
$$

for $i=1,2$. Next, we get from (48) and (50) the estimate

$$
\left|f_{i}\right|_{1}=O\left(n^{-1}\right)
$$


(47), (49), and Lemma 5 give us that

$$
\sup _{0 \leq \varphi \leq \max \left\{\hat{\varphi}_{n+1}^{\prime}, \tilde{\varphi}_{n+1}^{\prime}-\varphi_{n+1}^{\prime}+\hat{\delta}\right\}}\left|\hat{f}_{2}\right|>\frac{\Omega}{2}
$$

for large $n$; and it follows from the proof of Lemma 1 that

$$
\left|\tilde{\varphi}_{n+1}^{\prime}-\varphi_{n+1}^{\prime}\right|=O(1) \text {. }
$$

Substituting (56) and (54) in (53) and using (52) and Lemma 5, we obtain the estimate

$$
J^{(0)}=O\left(n^{-2}\right),
$$

which implies assertion 3) of Lemma 6. Substituting (57) in (51) and using (55), we get the estimate

$$
\left|\hat{\varphi}_{n+1}^{\prime}-\left(\tilde{\varphi}_{n+1}^{\prime}-\varphi_{n+1}^{\prime}\right)\right|=O\left(n^{-2}\right) .
$$

Let us now estimate $\left|\hat{z}_{n+1}^{\prime}-\tilde{z}_{n+1}^{\prime}\right|$. The quantity $\hat{z}_{n+1}^{\prime}-\tilde{z}_{n+1}^{\prime}$ is equal to

$$
\begin{aligned}
& \sqrt{\tilde{q}^{2}+\left(\hat{p}^{(n)}\left(\hat{\varphi}_{n+1}^{\prime}\right)+p_{n}^{\prime}\right)^{2}+1}-\sqrt{\tilde{q}^{2}+\left(p^{(n)}\left(\tilde{\varphi}_{n+1}^{\prime}-\varphi_{n+1}^{\prime}\right)+p_{n}^{\prime}\right)^{2}+1} \\
& =\frac{\left(\hat{p}^{(n)}\left(\hat{\varphi}_{n+1}^{\prime}\right)-p^{(n)}\left(\tilde{\varphi}_{n+1}^{\prime}-\varphi_{n+1}^{\prime}\right)\right)\left(\hat{p}^{(n)}\left(\hat{\varphi}_{n+1}^{\prime}\right)+p^{(n)}\left(\tilde{\varphi}_{n+1}^{\prime}-\varphi_{n+1}^{\prime}\right)+2 p_{n}^{\prime}\right)}{\sqrt{\tilde{q}^{2}+\left(\hat{p}^{(n)}\left(\hat{\varphi}_{n+1}^{\prime}\right)+p_{n}^{\prime}\right)^{2}+1}+\sqrt{\tilde{q}^{2}+\left(p^{(n)}\left(\tilde{\varphi}_{n+1}^{\prime}-\varphi_{n+1}^{\prime}\right)+p_{n}^{\prime}\right)^{2}+1}} .
\end{aligned}
$$

Therefore,

$$
\left|\hat{z}_{n+1}^{\prime}-\tilde{z}_{n+1}^{\prime}\right| \leq C_{5}\left|\hat{p}^{(n)}\left(\hat{\varphi}_{n+1}^{\prime}\right)-p^{(n)}\left(\tilde{\varphi}_{n+1}^{\prime}-\varphi_{n+1}^{\prime}\right)\right|,
$$

and by $(52),(57),(48)$, and (58) we get that

$$
\left|\hat{z}_{n+1}^{\prime}-\tilde{z}_{n+1}^{\prime}\right|=O\left(n^{-2}\right) .
$$

The estimates (58) and (59) prove assertion 2), and Lemma 6 is proved.

Lemma 7. For the initial data $\hat{p}^{(n)}(0)=0, \hat{q}^{(n)}(0)=0$ the solution of the system (47) has the form

$$
\begin{aligned}
& \hat{p}^{(n)}(\varphi)=\sqrt{\left(z^{(n)}(\varphi)+z_{n}+\tilde{z}\right)^{2}-1} \cos \left(\frac{\Omega\left(\varphi+\varphi_{n+1}^{\prime}-\tilde{\varphi}+\hat{\varphi}_{n}-\frac{2 \pi\left(z_{n}+\tilde{z}\right)}{\Omega}\right)}{z^{(n)}(\varphi)+z_{n}+\tilde{z}}\right)-p_{n}^{\prime}, \\
& \hat{q}^{(n)}(\varphi)=\sqrt{\left(z^{(n)}(\varphi)+z_{n}+\tilde{z}\right)^{2}-1} \sin \left(\frac{\Omega\left(\varphi+\varphi_{n+1}^{\prime}-\tilde{\varphi}+\hat{\varphi}_{n}-\frac{2 \pi\left(z_{n}+\tilde{z}\right)}{\Omega}\right)}{z^{(n)}(\varphi)+z_{n}+\tilde{z}}\right)-q_{n}^{\prime},
\end{aligned}
$$

where $p_{n}^{\prime}$ and $q_{n}^{\prime}$ are taken from Lemma 6 , and the function $z^{(n)}(\varphi)$ is taken from Definition 4.

Proof. We introduce the functions

$$
\begin{aligned}
& \tilde{p}^{(n)}(\varphi)=p_{n}^{\prime}+\hat{p}^{(n)}(\varphi), \\
& \tilde{q}^{(n)}(\varphi)=q_{n}^{\prime}+\hat{q}^{(n)}(\varphi), \\
& \tilde{z}^{(n)}(\varphi)=z_{n}+\tilde{z}+z^{(n)}(\varphi)
\end{aligned}
$$

and prove that if in the expressions for $\tilde{p}^{(n)}(\varphi)$ and $\tilde{q}^{(n)}(\varphi)$ we substitute the functions $\hat{p}^{(n)}(\varphi)$ and $\hat{q}^{(n)}(\varphi)$ in the statement of Lemma 7 , then the resulting $\tilde{p}^{(n)}(\varphi)$ and $\tilde{q}^{(n)}(\varphi)$ satisfy the system of equations

$$
\begin{aligned}
& \frac{d \tilde{p}^{(n)}}{d \varphi}=-\frac{\Omega \tilde{q}^{(n)}}{\sqrt{\left(\tilde{p}^{(n)}\right)^{2}+\left(\tilde{q}^{(n)}\right)^{2}+1}}+E\left(y\left(\varphi+\varphi_{n+1}^{\prime}\right), \varphi+\varphi_{n+1}^{\prime}\right), \\
& \frac{d \tilde{q}^{(n)}}{d \varphi}=\frac{\Omega \tilde{p}^{(n)}}{\sqrt{\left(\tilde{p}^{(n)}\right)^{2}+\left(\tilde{q}^{(n)}\right)^{2}+1}}+\Psi_{n}(\varphi),
\end{aligned}
$$


with $\tilde{p}^{(n)}(0)=p_{n}^{\prime}$ and $\tilde{q}^{(n)}(0)=q_{n}^{\prime}$. Indeed, from the definitions of $\tilde{z}^{(n)}(\varphi), \tilde{p}^{(n)}(\varphi)$, and $\tilde{q}^{(n)}(\varphi)$ we have the equalities

$$
\begin{gathered}
1+\left(\tilde{p}^{(n)}\right)^{2}+\left(\tilde{q}^{(n)}\right)^{2}=\left(\tilde{z}^{(n)}\right)^{2} \\
\tilde{p}^{(n)}(\varphi)=\sqrt{\left(\tilde{z}^{(n)}(\varphi)\right)^{2}-1} \cos \left(\frac{\Omega\left(\varphi+\varphi_{n+1}^{\prime}-\tilde{\varphi}+\hat{\varphi}_{n}-\frac{2 \pi\left(\tilde{z}+z_{n}\right)}{\Omega}\right)}{\tilde{z}^{(n)}}\right), \\
\tilde{q}^{(n)}(\varphi)=\sqrt{\left(\tilde{z}^{(n)}(\varphi)\right)^{2}-1} \sin \left(\frac{\Omega\left(\varphi+\varphi_{n+1}^{\prime}-\tilde{\varphi}+\hat{\varphi}_{n}-\frac{2 \pi\left(\tilde{z}+z_{n}\right)}{\Omega}\right)}{\tilde{z}^{(n)}}\right),
\end{gathered}
$$

and substituting these in (60), we get the relations

$$
\begin{aligned}
& \frac{d}{d \varphi}\left(\sqrt{\left(\tilde{z}^{(n)}(\varphi)\right)^{2}-1}\right) \cos \left(\frac{\Omega\left(\varphi+\varphi_{n+1}^{\prime}-\tilde{\varphi}+\hat{\varphi}_{n}-\frac{2 \pi\left(\tilde{z}+z_{n}\right)}{\Omega}\right)}{\tilde{z}^{(n)}}\right) \\
& \quad+\frac{\sqrt{\left(\tilde{z}^{(n)}\right)^{2}-1}}{\left(\tilde{z}^{(n)}\right)^{2}} \frac{d \tilde{z}^{(n)}}{d \varphi} \Omega\left(\varphi+\varphi_{n+1}^{\prime}-\tilde{\varphi}+\hat{\varphi}_{n}-\frac{2 \pi\left(\tilde{z}+z_{n}\right)}{\Omega}\right) \\
& \quad \times \sin \left(\frac{\Omega\left(\varphi+\varphi_{n+1}^{\prime}-\tilde{\varphi}+\hat{\varphi}_{n}-\frac{2 \pi\left(\tilde{z}+z_{n}\right)}{\Omega}\right)}{\tilde{z}^{(n)}}\right)=E\left(y\left(\varphi+\varphi_{n+1}^{\prime}\right), \varphi+\varphi_{n+1}^{\prime}\right), \\
& \frac{d}{d \varphi}\left(\sqrt{\left(\tilde{z}^{(n)}(\varphi)\right)^{2}+1}\right) \sin \left(\frac{\Omega\left(\varphi+\varphi_{n+1}^{\prime}-\tilde{\varphi}+\hat{\varphi}_{n}-\frac{2 \pi\left(\tilde{z}+z_{n}\right)}{\Omega}\right)}{\tilde{z}^{(n)}}\right) \\
& \left.\quad-\frac{\sqrt{\left(\tilde{z}^{(n)}\right)^{2}-1} \frac{d \tilde{z}^{(n)}}{d \varphi} \Omega\left(\varphi+\varphi_{n+1}^{\prime}-\tilde{\varphi}+\hat{\varphi}_{n}-\frac{2 \pi\left(\tilde{z}+z_{n}\right)}{\Omega}\right)}{\left(\tilde{z}^{(n)}\right)^{2}}\right) \\
& \quad \times \cos \left(\frac{\Omega\left(\varphi+\varphi_{n+1}^{\prime}-\tilde{\varphi}+\hat{\varphi}_{n}-\frac{2 \pi\left(\tilde{z}+z_{n}\right)}{\Omega}\right)}{\tilde{z}^{(n)}}\right)=\Psi_{n}(\varphi)
\end{aligned}
$$

which are valid in view of (41), (42), and (43).

Thus, the functions $\tilde{p}^{(n)}(\varphi)$ and $\tilde{q}^{(n)}(\varphi)$ satisfy the system (60), and hence the functions $\hat{p}^{(n)}(\varphi)$ and $\hat{q}^{(n)}(\varphi)$ satisfy the system $(47)$. Lemma 7 is proved.

Lemma 8. Let $\hat{\varphi}_{n+1}^{\prime}$ be the quantity defined in Lemma 6 , and let $\hat{\varphi}_{n+1, \infty}^{\prime}$ be such that

$$
\Omega\left(\hat{\varphi}_{n+1, \infty}^{\prime}+\varphi_{n+1}^{\prime}-\tilde{\varphi}+\hat{\varphi}_{n}-\frac{2 \pi\left(\tilde{z}+z_{n}\right)}{\Omega}\right)=\tilde{q} .
$$

Then

$$
\left|\hat{\varphi}_{n+1}^{\prime}-\hat{\varphi}_{n+1, \infty}^{\prime}\right|=O\left(n^{-2}\right) .
$$

Proof. By Lemma 7 and the definition of $\hat{\varphi}_{n+1}^{\prime}$,

$$
\begin{aligned}
\tilde{q}=q_{n}^{\prime}+\hat{q}^{(n)}\left(\hat{\varphi}_{n+1}^{\prime}\right)= & \sqrt{\left(z^{(n)}\left(\hat{\varphi}_{n+1}^{\prime}\right)+z_{n}+\tilde{z}\right)^{2}-1} \\
& \times \sin \left(\frac{\Omega\left(\hat{\varphi}_{n+1}^{\prime}+\varphi_{n+1}^{\prime}-\tilde{\varphi}+\hat{\varphi}_{n}-\frac{2 \pi\left(\tilde{z}+z_{n}\right)}{\Omega}\right)}{z^{(n)}\left(\hat{\varphi}_{n+1}^{\prime}\right)+z_{n}+\tilde{z}}\right) .
\end{aligned}
$$

Let

$$
\Phi_{n}(x)=\sqrt{\left(z^{(n)}(x)+z_{n}+\tilde{z}\right)^{2}-1} \sin \left(\frac{\Omega\left(x+\varphi_{n+1}^{\prime}-\tilde{\varphi}+\hat{\varphi}_{n}-\frac{2 \pi\left(\tilde{z}+z_{n}\right)}{\Omega}\right)}{z^{(n)}(x)+z_{n}+\tilde{z}}\right) .
$$

By Lemma 4, Lemma 2, and Definition 3,

$$
\lim _{n \rightarrow \infty} \Phi_{n}(x) \stackrel{\text { def }}{=} \Phi_{\infty}(x)=\Omega\left(x+\varphi_{\infty, n+1}^{\prime}-\tilde{\varphi}+\hat{\varphi}_{\infty}-\frac{2 \pi\left(\tilde{z}+z_{n}\right)}{\Omega}\right)=\Omega\left(x-2 b+\hat{\varphi}_{\infty}\right) .
$$


We prove that for $\hat{\delta}>0$

$$
\sup _{0 \leq x \leq \tilde{\varphi}_{n+1}^{\prime}-\varphi_{n+1}^{\prime}+\hat{\delta}}\left|\Phi_{n}(x)-\Phi_{\infty}(x)\right|=O\left(n^{-2}\right) .
$$

Indeed, according to Lemma 2 and Definition 3,

$$
\begin{aligned}
\left|\Phi_{n}(x)-\Phi_{\infty}(x)\right| \leq\left|\frac{\sqrt{\left(z^{(n)}(x)+z_{n}+\tilde{z}\right)^{2}-1}}{z^{(n)}(x)+z_{n}+\tilde{z}}-1\right| \\
\times\left|\Omega\left(x+\varphi_{n+1}^{\prime}-\tilde{\varphi}+\hat{\varphi}_{n}-\frac{2 \pi\left(\tilde{z}+z_{n}\right)}{\Omega}\right)\right|+O\left(n^{-2}\right),
\end{aligned}
$$

which obviously implies (62). Further, introducing the function

$$
\tilde{\Phi}_{\infty}(x) \stackrel{\text { def }}{=} \Omega\left(x+\varphi_{n+1}^{\prime}-\tilde{\varphi}+\hat{\varphi}_{n}-\frac{2 \pi\left(\tilde{z}+z_{n}\right)}{\Omega}\right),
$$

we get from (61) and the statement of Lemma 8 that

$$
\tilde{\Phi}_{\infty}\left(\hat{\varphi}_{n+1, \infty}^{\prime}\right)=\Phi_{n}\left(\hat{\varphi}_{\infty, n+1}^{\prime}\right)=\tilde{q} .
$$

Therefore, from Lemma 2, (62), and the definition of the functions $\Phi_{\infty}(x)$ and $\tilde{\Phi}_{\infty}(x)$ we have

$$
\begin{aligned}
& \left|\Omega\left(\hat{\varphi}_{n+1, \infty}^{\prime}-\hat{\varphi}_{n+1}^{\prime}\right)\right|=\left|\Phi_{\infty}\left(\hat{\varphi}_{n+1, \infty}^{\prime}\right)-\Phi_{\infty}\left(\hat{\varphi}_{n+1}^{\prime}\right)\right| \\
& \quad=\left|\tilde{\Phi}_{\infty}\left(\hat{\varphi}_{n+1, \infty}^{\prime}\right)+\left\{\Phi_{\infty}\left(\hat{\varphi}_{n+1, \infty}^{\prime}\right)-\tilde{\Phi}_{\infty}\left(\hat{\varphi}_{n+1, \infty}^{\prime}\right)\right\}-\Phi_{\infty}\left(\hat{\varphi}_{n+1}^{\prime}\right)\right| \\
& \quad \leq\left|\Phi_{n}\left(\hat{\varphi}_{n+1}^{\prime}\right)-\Phi_{\infty}\left(\hat{\varphi}_{n+1}^{\prime}\right)\right|+\left|\Phi_{\infty}\left(\hat{\varphi}_{n+1, \infty}^{\prime}\right)-\tilde{\Phi}_{\infty}\left(\hat{\varphi}_{n+1, \infty}^{\prime}\right)\right|=O\left(n^{-2}\right),
\end{aligned}
$$

which proves Lemma 8.

Lemma 9. Let

$$
\tilde{\varphi}_{\infty, n+1}^{\prime}=\tilde{\varphi}+\frac{2 \pi\left(\tilde{z}+z_{n}\right)}{\Omega}
$$

be the coordinate of the point $\left(\tilde{\varphi}_{\infty, n+1}^{\prime}, \tilde{z}_{\infty, n+1}^{\prime}\right)=\bar{A}_{\infty, n+1}(\tilde{\varphi}, \tilde{z})$ introduced in Definition 3 , and let $\hat{\varphi}_{n+1}^{\prime}$ be the quantity defined in Lemma 6 . Then

$$
\left|\varphi_{n+1}^{\prime}+\hat{\varphi}_{n+1}^{\prime}-\tilde{\varphi}_{\infty, n+1}^{\prime}\right|=O\left(n^{-2}\right)
$$

Proof. By Lemma 6,

$$
q_{n}^{\prime}=\sqrt{\left(\tilde{z}+z_{n}\right)^{2}-1} \sin \left(\frac{\Omega\left(\varphi_{n+1}^{\prime}-\tilde{\varphi}+\hat{\varphi}_{n}-\frac{2 \pi\left(\tilde{z}+z_{n}\right)}{\Omega}\right)}{\tilde{z}+z_{n}}\right) .
$$

Therefore, passing to the limit in the last equality, defining $q_{\infty}^{\prime}=\lim _{n \rightarrow \infty} q_{n}^{\prime}$, and using (15) and (16), we get that

$$
q_{\infty}^{\prime}=\lim _{n \rightarrow \infty} \Omega\left(\varphi_{n+1}^{\prime}-\tilde{\varphi}+\hat{\varphi}_{n}-\frac{2 \pi\left(\tilde{z}+z_{n}\right)}{\Omega}\right)=\Omega\left(\hat{\varphi}_{\infty}-2 b\right),
$$

where $\hat{\varphi}_{\infty}=\frac{\tilde{q}}{\Omega}$. Let

$$
\varphi_{n+1}^{\prime}-\tilde{\varphi}+\hat{\varphi}_{n}-\frac{2 \pi\left(\tilde{z}+z_{n}\right)}{\Omega}=\hat{\varphi}_{\infty}-2 b+\varepsilon_{n} .
$$

Then it follows from the definition of $\hat{\varphi}_{n+1, \infty}^{\prime}$ in Lemma 8, (63), and (64) that

$$
\Omega\left(\hat{\varphi}_{n+1, \infty}^{\prime}+\varepsilon_{n}\right)=\tilde{q}-q_{\infty}^{\prime} .
$$


Further, by (17) and the definition of the number $q^{*}=-\tilde{q}$ in this equality, we have the equality $\tilde{q}-q_{\infty}^{\prime}=2 b \Omega$, and if we substitute this in (65), we get that

$$
\hat{\varphi}_{n+1, \infty}^{\prime}+\varepsilon_{n}=2 b .
$$

Setting $\tilde{\varphi}_{n+1, \infty}^{\prime}-\varphi_{n+1}^{\prime}=2 b+\beta_{n}$, we get from (66) the equality

$$
\tilde{\varphi}_{n+1, \infty}^{\prime}-\varphi_{n+1}^{\prime}-\hat{\varphi}_{n+1, \infty}^{\prime}=\beta_{n}+\varepsilon_{n},
$$

and in view of Lemma 8, Lemma 9 will be proved if we prove that

$$
\varepsilon_{n}=O\left(n^{-2}\right), \quad \beta_{n}=O\left(n^{-2}\right) .
$$

According to Definition 3,

$$
\varphi_{\infty, n+1}^{\prime}=\tilde{\varphi}+\frac{2 \pi\left(\tilde{z}+z_{n}\right)}{\Omega}-2 b
$$

and

$$
\begin{aligned}
\varphi_{n+1}^{\prime} & -\tilde{\varphi}+\hat{\varphi}_{n}-\frac{2 \pi\left(\tilde{z}+z_{n}\right)}{\Omega} \\
& =\left(\varphi_{n+1}^{\prime}-\varphi_{\infty, n+1}^{\prime}\right)+\left(\hat{\varphi}_{n}-\hat{\varphi}_{\infty}\right)+\left(\varphi_{\infty, n+1}^{\prime}-\tilde{\varphi}+\hat{\varphi}_{\infty}-\frac{2 \pi\left(\tilde{z}+z_{n}\right)}{\Omega}\right) \\
& =\left(\varphi_{n+1}^{\prime}-\varphi_{\infty, n+1}^{\prime}\right)+\left(\hat{\varphi}_{n}-\hat{\varphi}_{\infty}\right)+\hat{\varphi}_{\infty}-2 b .
\end{aligned}
$$

Therefore, by (64), Lemma 2, and Definition 3,

$$
\left|\varepsilon_{n}\right| \leq\left|\varphi_{n+1}^{\prime}-\varphi_{\infty, n+1}^{\prime}\right|+\left|\hat{\varphi}_{n}-\hat{\varphi}_{\infty}\right|=O\left(n^{-2}\right) .
$$

Then it follows from Definition 3 that

$$
\tilde{\varphi}_{\infty, n+1}^{\prime}-\varphi_{n+1}^{\prime}=\tilde{\varphi}_{\infty, n+1}^{\prime}-\varphi_{\infty, n+1}^{\prime}+\left(\varphi_{\infty, n+1}^{\prime}-\varphi_{n+1}^{\prime}\right)=2 b+\left(\varphi_{\infty, n+1}^{\prime}-\varphi_{n+1}^{\prime}\right) .
$$

Therefore, according to Lemma 2 ,

$$
\left|\beta_{n}\right|=\left|\varphi_{\infty, n+1}^{\prime}-\varphi_{n+1}^{\prime}\right|=O\left(n^{-2}\right) .
$$

This estimate and (68) prove (67) and thereby Lemma 9.

Lemma 10. Let $\tilde{\varphi}_{n+1}>0$ be the number closest to zero such that

$$
\tilde{q}=\sqrt{\left(\tilde{z}+z_{n}\right)^{2}-1} \sin \left(\frac{\Omega\left(\varphi_{n+1}^{\prime}+\tilde{\varphi}_{n+1}-\tilde{\varphi}+\hat{\varphi}_{n}-\frac{2 \pi\left(\tilde{z}+z_{n}\right)}{\Omega}\right)}{\tilde{z}+z_{n}}\right),
$$

and let $\varphi_{n+1, \infty}^{\prime}$ be the number defined in Lemma 8. Then

$$
\left|\tilde{\tilde{\varphi}}_{n+1}-\hat{\varphi}_{n+1, \infty}^{\prime}\right|=O\left(n^{-2}\right) \text {. }
$$

Proof. Let

$$
\tilde{\tilde{\Phi}}_{n}(x)=\sqrt{\left(\tilde{z}+z_{n}\right)^{2}-1} \sin \left(\frac{\Omega\left(x+\varphi_{n+1}^{\prime}-\tilde{\varphi}+\hat{\varphi}_{n}-\frac{2 \pi\left(\tilde{z}+z_{n}\right)}{\Omega}\right)}{\tilde{z}+z_{n}}\right) .
$$

Repeating the proof of Lemma 8 with the function $\Phi_{n}(x)$ in it replaced by $\tilde{\tilde{\Phi}}_{n}(x)$, we obtain Lemma 10.

Lemma 11.

$$
\left|\tilde{x}-x\left(\varphi_{n+1}^{\prime}+\tilde{\tilde{\varphi}}_{n+1}\right)\right|=O\left(n^{-2}\right),
$$

where $\tilde{\tilde{\varphi}}_{n+1}$ is the quantity defined in Lemma 10. 
Proof. By (1),

$$
x\left(\varphi+\varphi_{n+1}^{\prime}\right)=x\left(\varphi_{n+1}^{\prime}\right)+\int_{\varphi_{n+1}^{\prime}}^{\varphi_{n+1}^{\prime}+\varphi} v(\tau) d \tau,
$$

and it follows from (3) that

$$
v=\frac{q}{\sqrt{1+p^{2}+q^{2}}}
$$

If we now substitute the equality (70) in (69), we get that

$$
\begin{aligned}
x(\varphi+ & \left.\varphi_{n+1}^{\prime}\right)=x\left(\varphi_{n+1}^{\prime}\right) \\
& +\int_{\varphi_{n+1}^{\prime}}^{\varphi_{n+1}^{\prime}+\varphi} \frac{\left(\hat{q}^{(n)}\left(\tau-\varphi_{n+1}^{\prime}\right)+q_{n}^{\prime}\right) d \tau}{\sqrt{1+\left(p_{n}^{\prime}+\hat{p}^{(n)}\left(\tau-\varphi_{n+1}^{\prime}\right)\right)^{2}+\left(q_{n}^{\prime}+\hat{q}^{(n)}\left(\tau-\varphi_{n+1}^{\prime}\right)\right)^{2}}} \\
& +\int_{\varphi_{n+1}^{\prime}}^{\varphi_{n+1}^{\prime}+\varphi} d \tau\left\{\frac{q(\tau)}{\sqrt{1+p^{2}(\tau)+q^{2}(\tau)}}\right. \\
& \left.-\frac{\hat{q}^{(n)}\left(\tau-\varphi_{n+1}^{\prime}\right)+q_{n}^{\prime}}{\sqrt{1+\left(p_{n}^{\prime}+\hat{p}^{(n)}\left(\tau-\varphi_{n+1}^{\prime}\right)\right)^{2}+\left(q_{n}^{\prime}+\hat{q}^{(n)}\left(\tau-\varphi_{n+1}^{\prime}\right)\right)^{2}}}\right\} \\
= & x\left(\varphi_{n+1}^{\prime}\right)+I_{1}+I_{2},
\end{aligned}
$$

where $I_{1}=I_{1}(\varphi)$ is the first integral, $I_{2}=I_{2}(\varphi)$ is the second, and the functions $\hat{p}^{(n)}$ and $\hat{q}^{(n)}$ and the numbers $p_{n}^{\prime}$ and $q_{n}^{\prime}$ are taken from Lemma 6 . It follows from Lemma 7 that the integral $I_{1}\left(\tilde{\tilde{\varphi}}_{n+1}\right)$ is

$$
\begin{aligned}
I_{1}\left(\tilde{\varphi}_{n+1}\right)=\int_{\varphi_{n+1}^{\prime}}^{\varphi_{n+1}^{\prime}+\tilde{\varphi}_{n+1}} \frac{\sqrt{\left(z^{(n)}\left(\tau-\varphi_{n+1}^{\prime}\right)+\tilde{z}+z_{n}\right)^{2}-1}}{z^{(n)}\left(\tau-\varphi_{n+1}^{\prime}\right)+\tilde{z}+z_{n}} \\
\quad \times \sin \left(\frac{\Omega\left(\tau-\varphi_{n+1}^{\prime}+\varphi_{n+1}^{\prime}-\tilde{\varphi}+\hat{\varphi}_{n}-\frac{2 \pi\left(\tilde{z}+z_{n}\right)}{\Omega}\right)}{z^{(n)}\left(\tau-\varphi_{n+1}^{\prime}\right)+\tilde{z}+z_{n}}\right) d \tau,
\end{aligned}
$$

and it follows from the definition of the number $\tilde{\tilde{\varphi}}_{n+1}$ in Lemma 10 together with (69), (70), (5), and (7) that

$$
\begin{array}{rl}
\tilde{x}=x\left(\varphi_{n+1}^{\prime}\right)+\int_{\varphi_{n+1}^{\prime}}^{\varphi_{n+1}^{\prime}+\tilde{\varphi}_{n+1}} & d \tau \frac{\sqrt{\left(\tilde{z}+z_{n}\right)^{2}-1}}{\tilde{z}+z_{n}} \\
& \times \sin \left(\frac{\Omega\left(\tau-\varphi_{n+1}^{\prime}+\varphi_{n+1}^{\prime}-\tilde{\varphi}+\hat{\varphi}_{n}-\frac{2 \pi\left(\tilde{z}+z_{n}\right)}{\Omega}\right)}{\tilde{z}+z_{n}}\right) .
\end{array}
$$


Therefore, by Lemma 4 and the last two equalities,

$$
\begin{aligned}
& \left|\tilde{x}-x\left(\varphi_{n+1}^{\prime}\right)-I_{1}\left(\tilde{\tilde{\varphi}}_{n+1}\right)\right| \\
& \leq C_{6} \cdot \sup _{\varphi_{n+1}^{\prime} \leq \tau \leq \varphi_{n+1}^{\prime}+\tilde{\varphi}_{n+1}} \mid\left(\frac{\sqrt{\left(z^{(n)}\left(\tau-\varphi_{n+1}^{\prime}\right)+\tilde{z}+z_{n}\right)^{2}-1}}{z^{(n)}\left(\tau-\varphi_{n+1}^{\prime}\right)+\tilde{z}+z_{n}}-1\right) \\
& \quad-\left(\frac{\sqrt{\left.\tilde{z}+z_{n}\right)^{2}-1}}{\tilde{z}+z_{n}}-1\right) \mid \\
& \quad C_{7} \cdot \sup _{\varphi_{n+1}^{\prime} \leq \tau \leq \varphi_{n+1}^{\prime}+\tilde{\varphi}_{n+1}} \mid \sin \left(\frac{\Omega\left(\tau-\tilde{\varphi}+\hat{\varphi}_{n}-\frac{2 \pi\left(\tilde{z}+z_{n}\right)}{\Omega}\right)}{z^{(n)}\left(\tau-\varphi_{n+1}^{\prime}\right)+\tilde{z}+z_{n}}\right) \\
&
\end{aligned}
$$

Further, in view of the equalities

$$
\begin{aligned}
& p(\tau)=p_{n}^{\prime}+\hat{p}^{(n)}\left(\tau-\varphi_{n+1}^{\prime}\right)+\left\{p^{(n)}\left(\tau-\varphi_{n+1}^{\prime}\right)-\hat{p}^{(n)}\left(\tau-\varphi_{n+1}^{\prime}\right)\right\}, \\
& q(\tau)=q_{n}^{\prime}+\hat{q}^{(n)}\left(\tau-\varphi_{n+1}^{\prime}\right)+\left\{q^{(n)}\left(\tau-\varphi_{n+1}^{\prime}\right)-\hat{q}^{(n)}\left(\tau-\varphi_{n+1}^{\prime}\right)\right\}
\end{aligned}
$$

and the assertion 3) in Lemma 6 we have the estimate $\left|I_{2}\left(\tilde{\tilde{\varphi}}_{n+1}\right)\right|=O\left(n^{-2}\right)$. Using the last two estimates and the equality (71), we now obtain the assertion of Lemma 11.

Lemma 12. $\left|\tilde{x}-x\left(\tilde{\varphi}_{n+1}^{\prime}\right)\right|=O\left(n^{-2}\right)$.

Proof. By Lemma 11, it suffices to prove that

$$
\left|x\left(\varphi_{n+1}^{\prime}+\tilde{\tilde{\varphi}}_{n+1}\right)-x\left(\tilde{\varphi}_{n+1}^{\prime}\right)\right|=O\left(n^{-2}\right) .
$$

We have

$$
\left|x\left(\varphi_{n+1}^{\prime}+\tilde{\tilde{\varphi}}_{n+1}\right)-x\left(\tilde{\varphi}_{n+1}^{\prime}\right)\right|=\left|\int_{\varphi_{n+1}^{\prime}+\tilde{\varphi}_{n+1}}^{\tilde{\varphi}_{n+1}^{\prime}} v(\tau) d \tau\right| .
$$

Therefore, by (70),

$$
\left|x\left(\varphi_{n+1}^{\prime}+\tilde{\tilde{\varphi}}_{n+1}\right)-x\left(\tilde{\varphi}_{n+1}^{\prime}\right)\right| \leq C_{8}\left|\tilde{\varphi}_{n+1}^{\prime}-\left(\varphi_{n+1}^{\prime}+\tilde{\tilde{\varphi}}_{n+1}\right)\right| .
$$

Further,

$$
\begin{aligned}
\tilde{\varphi}_{n+1}^{\prime}-\left(\varphi_{n+1}^{\prime}+\tilde{\tilde{\varphi}}_{n+1}\right)= & \hat{\varphi}_{n+1}^{\prime}-\tilde{\varphi}_{n+1}+\left\{\left(\tilde{\varphi}_{n+1}^{\prime}-\varphi_{n+1}^{\prime}\right)-\hat{\varphi}_{n+1}^{\prime}\right\} \\
= & \left(\hat{\varphi}_{n+1}^{\prime}-\hat{\varphi}_{n+1, \infty}^{\prime}\right)+\left(\hat{\varphi}_{n+1, \infty}^{\prime}-\tilde{\tilde{\varphi}}_{n+1}\right) \\
& +\left\{\left(\tilde{\varphi}_{n+1}^{\prime}-\varphi_{n+1}^{\prime}\right)-\hat{\varphi}_{n+1}^{\prime}\right\} .
\end{aligned}
$$

Therefore, $\left|\tilde{\varphi}_{n+1}^{\prime}-\left(\varphi_{n+1}^{\prime}+\tilde{\tilde{\varphi}}_{n+1}\right)\right|=O\left(n^{-2}\right)$ in view of Lemmas 6 , 8, and 10, and substitution of this estimate in (72) gives us Lemma 12.

Lemma 13. Let $y(s)$ be the solution of the equation

$$
\frac{d y}{d s}=\frac{p(s)}{\sqrt{q^{2}(s)+p^{2}(s)+1}}
$$

with initial data $y\left(\varphi_{n+1}^{\prime}\right)=-b$, where $p\left(\varphi_{n+1}^{\prime}\right)=p_{n}^{\prime}, q\left(\varphi_{n+1}^{\prime}\right)=q_{n}^{\prime}$, and the numbers $p_{n}^{\prime}$ and $q_{n}^{\prime}$ are taken from Lemma 6 . Then

$$
\sup _{\varphi_{n+1}^{\prime} \leq s \leq \tilde{\varphi}_{n+1}^{\prime}+\hat{\delta}}\left|y(s)-\left(s-\varphi_{n+1}^{\prime}-b\right)\right|=O\left(n^{-2}\right),
$$

where $\hat{\delta}>0$ is a constant independent of $n, \tilde{q}, \tilde{x}, \tilde{\varphi}, \tilde{z}$. 
Proof. By a condition of Lemma 13 together with Lemma 6,

$$
y(s)=-b+\int_{\varphi_{n+1}^{\prime}}^{s}\left(I_{1}^{\prime}(\tau)+I_{2}^{\prime}(\tau)\right) d \tau
$$

for $s \geq \varphi_{n+1}^{\prime}$, where

$$
\begin{aligned}
I_{1}^{\prime}(\tau)= & \frac{\hat{p}^{(n)}(\tau)+p_{n}^{\prime}}{\sqrt{1+\left(q_{n}^{\prime}+\hat{q}^{(n)}\left(\tau-\varphi_{n+1}^{\prime}\right)\right)^{2}+\left(p_{n}^{\prime}+\hat{p}^{(n)}\left(\tau-\varphi_{n+1}^{\prime}\right)\right)^{2}}}, \\
I_{2}^{\prime}(\tau)= & \frac{p(\tau)}{\sqrt{1+p^{2}(\tau)+q^{2}(\tau)}} \\
& -\frac{\hat{p}^{(n)}(\tau)+p_{n}^{\prime}}{\sqrt{1+\left(q_{n}^{\prime}+\hat{q}^{(n)}\left(\tau-\varphi_{n+1}^{\prime}\right)\right)^{2}+\left(p_{n}^{\prime}+\hat{p}^{(n)}\left(\tau-\varphi_{n+1}^{\prime}\right)\right)^{2}}} .
\end{aligned}
$$

It follows from Lemma 7 that $I_{1}^{\prime}$ has the form

$$
\begin{aligned}
I_{1}^{\prime}(\tau)= & \frac{\sqrt{\left(z^{(n)}\left(\tau-\varphi_{n+1}^{\prime}\right)+\tilde{z}+z_{n}\right)^{2}-1}}{z^{(n)}\left(\tau-\varphi_{n+1}^{\prime}\right)+\tilde{z}+z_{n}} \\
& \times \cos \left(\frac{\Omega\left(\tau-\varphi_{n+1}^{\prime}+\varphi_{n+1}^{\prime}-\tilde{\varphi}+\hat{\varphi}_{n}-\frac{2 \pi\left(\tilde{z}+z_{n}\right)}{\Omega}\right)}{z^{(n)}\left(\tau-\varphi_{n+1}^{\prime}\right)+\tilde{z}+z_{n}}\right) .
\end{aligned}
$$

Therefore, by Definition 2 and Lemmas 2 and 4 ,

$$
\begin{aligned}
& \sup _{\varphi_{n+1}^{\prime} \leq \tau \leq \tilde{\varphi}_{n+1}^{\prime}+\hat{\delta}}\left|I_{1}^{\prime}(\tau)-1\right| \leq \sup _{\varphi_{n+1}^{\prime} \leq \tau \leq \tilde{\varphi}_{n+1}^{\prime}+\delta}\left|\frac{\sqrt{\left(z^{(n)}\left(\tau-\varphi_{n+1}^{\prime}\right)+\tilde{z}+z_{n}\right)^{2}-1}}{z^{(n)}\left(\tau-\varphi_{n+1}^{\prime}\right)+\tilde{z}+z_{n}}-1\right| \\
& +\sup _{\varphi_{n+1}^{\prime} \leq \tau \leq \tilde{\varphi}_{n+1}^{\prime}+\delta}\left|\cos \left(\frac{\Omega\left(\tau-\varphi_{n+1}^{\prime}+\left(\varphi_{n+1}^{\prime}-\varphi_{\infty, n+1}^{\prime}\right)+\varphi_{n}^{\prime}-2 b\right)}{z^{(n)}\left(\tau-\varphi_{n+1}^{\prime}\right)+\tilde{z}+z_{n}}\right)-1\right|=O\left(n^{-2}\right),
\end{aligned}
$$

and by assertion 3 ) of Lemma 6 ,

$$
\sup _{\varphi_{n+1}^{\prime} \leq \tau \leq \tilde{\varphi}_{n+1}^{\prime}+\hat{\delta}}\left|I_{2}^{\prime}(\tau)\right|=O\left(n^{-2}\right) .
$$

By (73),

$$
y(s)-\left(s-\varphi_{n+1}^{\prime}-b\right)=\int_{\varphi_{n+1}^{\prime}}^{s}\left(\left(I_{1}^{\prime}(s)-1\right)+I_{2}^{\prime}(s)\right) d s,
$$

hence substitution of the last two estimates in this equality gives us Lemma 13.

Lemma 14. Let $\left(\tilde{\varphi}_{\infty, n+1}^{\prime}, \tilde{z}_{\infty, n+1}^{\prime}\right)=\bar{A}_{\infty, n+1}(\tilde{\varphi}, \tilde{z})$ be the point introduced in Definition 3. Then

$$
\left|\tilde{\varphi}_{n+1}^{\prime}-\tilde{\varphi}_{\infty, n+1}^{\prime}\right|+\left|\tilde{z}_{n+1}^{\prime}-\tilde{z}_{\infty, n+1}^{\prime}\right|=O\left(n^{-2}\right) .
$$

Proof. By Lemma 9, $\varphi_{n+1}^{\prime}+\hat{\varphi}_{n+1}^{\prime}-\tilde{\varphi}_{\infty, n+1}^{\prime}=O\left(n^{-2}\right)$, and according to Lemma 6 we have $\hat{\varphi}_{n+1}^{\prime}+\varphi_{n+1}^{\prime}-\tilde{\varphi}_{n+1}^{\prime}=O\left(n^{-2}\right)$. Therefore, subtracting the first equality from the second, we get that

$$
\left|\tilde{\varphi}_{n+1}^{\prime}-\tilde{\varphi}_{\infty, n+1}^{\prime}\right|=O\left(n^{-2}\right) .
$$

By assertion 2) of Lemma $6,\left|\hat{z}_{n+1}^{\prime}-\tilde{z}_{n+1}^{\prime}\right|=O\left(n^{-2}\right)$. Therefore, to prove Lemma 14 it suffices to prove that

$$
\left|\hat{z}_{n+1}^{\prime}-\tilde{z}_{\infty, n+1}^{\prime}\right|=O\left(n^{-2}\right)
$$


According to Lemmas 6 and 7 ,

$$
\hat{z}_{n+1}^{\prime}=z_{n}+\tilde{z}+z^{(n)}\left(\hat{\varphi}_{n+1}^{\prime}\right),
$$

where $z^{(n)}(\varphi)$ is the function introduced in Definition 4 , and it follows from Lemma 4 that

$$
\left|z^{(n)}\left(\hat{\varphi}_{n+1}^{\prime}\right)-\int_{\varphi_{\infty, n+1}^{\prime}}^{\varphi_{\infty, n+1}^{\prime}+\hat{\varphi}_{n+1}^{\prime}} E(y(s), s) d s\right|=O\left(n^{-2}\right) .
$$

In view of Lemmas 9 and 2

$$
\left|\tilde{\varphi}_{\infty, n+1}^{\prime}-\hat{\varphi}_{n+1}^{\prime}-\varphi_{\infty, n+1}^{\prime}\right|=O\left(n^{-2}\right)
$$

thus,

$$
\left|\int_{\varphi_{\infty, n+1}^{\prime}}^{\varphi_{\infty, n+1}^{\prime}+\hat{\varphi}_{n+1}^{\prime}} E(y(s), s) d s-\int_{\varphi_{\infty, n+1}^{\prime}}^{\tilde{\varphi}_{\infty, n+1}^{\prime}} E(y(s), s) d s\right|=O\left(n^{-2}\right) .
$$

Therefore, by Definition 3 of the quantities $\tilde{z}_{\infty, n+1}^{\prime}, \tilde{\varphi}_{\infty, n+1}^{\prime}$, and $\varphi_{\infty, n+1}^{\prime}$ together with the equalities (76), (77), and (78), to prove (75) it suffices to prove the estimate

$$
\left|E(y(s), s)-E\left(s-\varphi_{\infty, n+1}^{\prime}-b, s\right)\right|=O\left(n^{-2}\right),
$$

which is a consequence of Lemmas 13 and 2. Thus, the estimate (75) and with it Lemma 14 is proved.

Lemma 15. Let

$$
\begin{gathered}
\bar{A}_{n}:(\tilde{\varphi}, \tilde{z}) \longmapsto\left(\tilde{\varphi}_{n}^{\prime}, \tilde{z}_{n}^{\prime}\right) \stackrel{\text { def }}{=}\left(f^{(n)}(\tilde{\varphi}, \tilde{z}), g^{(n)}(\tilde{\varphi}, \tilde{z})\right), \\
\bar{A}_{\infty, n}:(\tilde{\varphi}, \tilde{z}) \longmapsto\left(\tilde{\varphi}_{\infty, n}^{\prime}, \tilde{z}_{\infty, n}^{\prime}\right) \stackrel{\text { def }}{=}\left(f^{(\infty)}(\tilde{\varphi}, \tilde{z}), g^{(\infty)}(\tilde{\varphi}, \tilde{z})\right) .
\end{gathered}
$$

Then for any $\varepsilon>0$ there is an $n_{0}>0$ independent of $\tilde{q}$ and $\tilde{x}$ such that for $n \geq n_{0}$, $-\varkappa \leq \tilde{q}-l \leq \varkappa$, and $|x| \leq \hat{\varepsilon}$

$$
\left|f^{(n)}(\tilde{\varphi}, \tilde{z})-f^{(\infty)}(\tilde{\varphi}, \tilde{z})\right|_{1}+\left|g^{(n)}(\tilde{\varphi}, \tilde{z})-g^{(\infty)}(\tilde{\varphi}, \tilde{z})\right|_{1} \leq \varepsilon,
$$

where for an arbitrary differentiable function $f(\tilde{\varphi}, \tilde{z})$

$$
|f(\tilde{\varphi}, \tilde{z})|_{1}=\sup _{(\tilde{\varphi}, \tilde{z}) \in \mathcal{U}_{\varepsilon_{0}}} \max \left\{\left|\frac{\partial f}{\partial \tilde{\varphi}}(\tilde{\varphi}, \tilde{z})\right|,\left|\frac{\partial f}{\partial \tilde{z}}(\tilde{\varphi}, \tilde{z})\right|\right\},
$$

and $\mathcal{U}_{\varepsilon_{0}}$ is the domain introduced in Lemma 2.

Proof. We define the function $\Delta^{(n)}=\Delta^{(n)}(\tilde{\varphi}, \tilde{z})$ by means of the equality

$$
\varphi_{n}^{\prime}=\tilde{\varphi}+\frac{2 \pi\left(\tilde{z}+z_{n-1}\right)}{\Omega}-2 b+\frac{2 \tilde{q}}{\Omega}-2 \hat{\varphi}_{n-1}+\Delta^{(n)},
$$

where $\varphi_{n}^{\prime}$ is the coordinate of the point $\left(\varphi_{n}^{\prime}, z_{n}^{\prime}\right)=\bar{A}_{n}^{\prime}(\tilde{\varphi}, \tilde{z})$. Applying to the systems (23), (24) the theorem on the continuously differentiable dependence on the parameter and the initial data, we get in view of (25) and (17) that as $n \rightarrow \infty$ the quantity $\tilde{\varphi}_{n}^{\prime}-\varphi_{n}^{\prime}$ together with the first derivatives with respect to $\tilde{q}$ and $\tilde{z}$ converges uniformly to the quantity

$$
\frac{1}{\Omega}\left(\tilde{q}-q^{*}+2 b \Omega-2 \tilde{q}\right)=2 b .
$$

Therefore as $n \rightarrow \infty$ the quantity $\tilde{\varphi}_{\infty, n}^{\prime}-\tilde{\varphi}_{n}^{\prime}$ together with the first derivatives converges uniformly to the quantity

$$
\lim _{n \rightarrow \infty}\left(\varphi_{\infty, n}^{\prime}-\varphi_{n}^{\prime}\right)-2 b,
$$


which by Definition 3, (79), (16), (15), (14), and (6) is

$$
\lim _{n \rightarrow \infty}\left(2 \hat{\varphi}_{n-1}-\frac{2 \tilde{q}}{\Omega}-\Delta^{(n)}\right)=0 .
$$

Similarly, using (5), (18), (24), (25), and the equality $z=\sqrt{p^{2}+q^{2}+1}$, which follows from (3), we can prove that as $n \rightarrow \infty$ the quantity $\tilde{z}_{\infty, n}^{\prime}-\tilde{z}_{n}^{\prime}$ together with the first derivatives converges uniformly to zero. Lemma 15 is proved.

Lemma 16. The system (1), (2) has the first integral $I=\Omega y-q$.

The proof clearly follows from (1), (2) and the notation in (3).

Lemma 17. Suppose that

$$
\begin{gathered}
|\tilde{x}|<\hat{\varepsilon}<a, \quad \tilde{q}=\frac{\tilde{v}}{\sqrt{1-\tilde{u}^{2}-\tilde{v}^{2}}}, \quad|\tilde{q}-l| \leq \varkappa, \quad \varphi_{0}=\frac{l}{\Omega}, \quad z_{0}=m \Omega, \\
\varphi_{n}=\varphi_{0}+2 \pi(m+k(n-1)), \quad z_{n}=z_{0}+n k \Omega,
\end{gathered}
$$

where $l>0, m$ is a positive integer, and $\varphi_{0}$ and $k$ are numbers satisfying the condition of the Basic Theorem. Then for any $\varepsilon>0$ there exist a positive integer $n_{0}=n_{0}(\varepsilon)$ and a number $\delta>0$ such that if

$$
(\varphi, z) \in \mathcal{U}_{\delta}^{\left(n_{0}\right)} \stackrel{\text { def }}{=}\left\{\varphi, z:\left|\varphi-\varphi_{n_{0}}\right| \leq \delta,\left|z-z_{n_{0}}\right| \leq \delta\right\},
$$

then for all $n=1,2, \ldots$ the points $\left(\varphi^{(n)}, z^{(n)}\right)=A^{n}(\varphi, z)$ are defined ( $A^{n}$ is the $n$th power of $A$ ) and

$$
\left(\varphi^{(n)}, z^{(n)}\right) \in \mathcal{U}_{\varepsilon}^{\left(n_{0}+n\right)} \stackrel{\text { def }}{=}\left\{\varphi, z:\left|\varphi-\varphi_{n_{0}+n}\right| \leq \varepsilon,\left|z-z_{n_{0}+n}\right| \leq \varepsilon\right\} .
$$

Proof. Let $\delta \leq \varepsilon_{0}$, where $\varepsilon_{0}$ is the constant introduced in Lemma 2. We consider the transformation

$$
A_{\infty}:(\varphi, z) \longmapsto\left(\varphi_{\infty}, z_{\infty}\right) \stackrel{\text { def }}{=}\left(\varphi+\frac{2 \pi z}{\Omega}, z+\Delta\left(\varphi_{\infty}\right)\right)
$$

$(\Delta(\varphi)$ is the function introduced in Definition 2) and the sequence of points

$$
\left(\varphi_{n}^{0}, z_{n}^{0}\right)=A_{\infty}^{n}\left(\varphi_{0}, z_{0}\right), \quad n=1,2, \ldots
$$

According to the conditions of Lemma 17 and Definitions 2 and 3 , for $n=1,2, \ldots$

and

$$
z_{n}^{0}=z_{n}, \quad \varphi_{n}^{0}=\varphi_{n}, \quad \varphi_{0} \equiv \varphi_{1}^{0} \equiv \cdots \equiv \varphi_{n}^{0} \equiv \cdots \bmod 2 \pi
$$

We now introduce the transformation $\tilde{A}$ by

$$
\begin{aligned}
\tilde{A}(\varphi, z) & \stackrel{\text { def }}{=} A_{\infty}\left(\varphi+\varphi_{n-1}^{0}, z+z_{n-1}^{0}\right)-\left(\varphi_{n}^{0}, z_{n}^{0}\right) \\
& \stackrel{\text { def }}{=}\left(\tilde{\varphi}_{\infty}, \tilde{z}_{\infty}\right)=\left(\varphi+\frac{2 \pi z}{\Omega}, z+\Delta\left(\tilde{\varphi}_{\infty}\right)-\Delta\left(\varphi_{0}\right)\right),
\end{aligned}
$$

and the sequence of transformations

$$
\tilde{B}_{n}:(\varphi, z) \longmapsto\left(\tilde{\varphi}_{n}, \tilde{z}_{n}\right) \stackrel{\text { def }}{=}(\varphi, z)+\bar{A}_{n}\left(\varphi+\varphi_{0}, z\right)-\bar{A}_{\infty, n}\left(\varphi+\varphi_{0}, z\right)
$$

$(n=1,2, \ldots)$, where $\bar{A}_{n}$ and $\bar{A}_{\infty, n}$ are the transformations in Definition 3. From (80)(84) it follows easily that $\tilde{A}$ does not depend on $n$, and if $n_{0}$ is a positive integer, then for any $n$

$A^{n}\left(\varphi+\varphi_{n_{0}}, z+z_{n_{0}}\right)-\bar{A}_{\infty, n}^{n}\left(\varphi_{n_{0}}, z_{n_{0}}\right)=\tilde{B}_{n_{0}+n} \circ \tilde{A} \circ B_{n_{0}+n-1} \circ \tilde{A} \circ \cdots \circ \tilde{B}_{n_{0}+1} \circ \tilde{A}(\varphi, z)$

if the points $A^{n}\left(\varphi+\varphi_{n_{0}}, z+z_{n_{0}}\right)$ are defined. 
By the last equality together with (80), (81), and (82), to prove Lemma 17 it suffices to prove that for any $\varepsilon>0$ there exist $\delta>0$ and $n_{0}=n_{0}(\varepsilon)$ such that if

$$
(\varphi, z) \in \tilde{\mathcal{U}}_{\delta} \stackrel{\text { def }}{=}\{\varphi, z:|\varphi|<\delta,|z|<\delta\}, \quad|\tilde{q}-l| \leq \varkappa, \quad|\tilde{x}|<\hat{\varepsilon},
$$

then for all $n \geq 1$ the points $A^{n}\left(\varphi+\varphi_{n_{0}}, z+z_{n_{0}}\right)$ are defined and

$$
\tilde{B}_{n_{0}+n} \circ \tilde{A} \circ B_{n_{0}+n-1} \circ \tilde{A} \circ \cdots \circ \tilde{B}_{n_{0}+1} \circ \tilde{A}(\varphi, z) \in \tilde{\mathcal{U}}_{\varepsilon} .
$$

To prove (85) we first assume for $n \geq 1$ that $(\varphi, z) \in \tilde{\mathcal{U}}_{\delta},|\tilde{q}-l| \leq \varkappa,|\tilde{x}|<\hat{\varepsilon}$, and the points $A^{n}\left(\varphi+\varphi_{n_{0}}, z+z_{n_{0}}\right)$ are defined, and we prove that for large $n_{0}$ the two sequences of transformations $A_{n}=\tilde{A}$ and $B_{n}=\tilde{B}_{n_{0}+n}$ satisfy in $\tilde{\mathcal{U}}_{\delta}$ all the conditions of Theorem 1 in $\S 2$ of [6] and Theorem 2 in $\S 1, \mathrm{Ch} . \mathrm{V}$ of [7], which in our case reduce to the following assertions.

1. $A_{n}$ is defined for $n=1,2, \ldots$ in the complex domain

$$
\mathcal{U}_{\delta}^{*}=\{\varphi, z: \max \{|\varphi|,|z|\} \leq \delta\} .
$$

2. $\tilde{A}$ is a transformation of general elliptic type, and in its Birkhoff normal form [16]

$$
\alpha_{1}=\alpha+\omega_{0}+\omega_{1} r+\cdots+\omega_{k} r^{k}+\cdots, \quad r_{1}=r
$$

( $\alpha, r$ are polar coordinates), with the coefficient $\omega_{1}$ nonzero (it follows from the definition of $\tilde{A}$ that $\tilde{A}$ is area preserving).

3. The coefficient $\omega_{0}$ satisfies the inequalities

$$
\omega_{0} \neq 2 \pi \frac{t}{\nu}, \quad \text { where } \quad t=0, \pm 1, \ldots, \pm \nu, \quad \nu=1,2,3, \ldots, l_{0}, \quad l_{0} \geq 4 .
$$

4. $B_{n}$ is a homeomorphism of the real domain $\operatorname{Re} \mathcal{U}_{\delta}^{*}$ and has the form

$$
B_{n}:(\varphi, z) \longmapsto\left(\tilde{\varphi}_{n}, \tilde{z}_{n}\right)=\left(\varphi+b_{1}^{(n)}(\varphi, z), z+b_{2}^{(n)}(\varphi, z)\right),
$$

and

$$
\sum_{n=1}^{\infty} \sup _{(\varphi, z) \in \operatorname{Re} \mathcal{U}_{\delta}^{*}}\left(\left|b_{1}^{(n)}(\varphi, z)\right|+\left|b_{2}^{(n)}(\varphi, z)\right|\right)<\infty .
$$

The assertion 1 is obvious, the assertion 3 follows from the condition 2) of Theorem 1 , and the assertion 2 follows from a condition of the Basic Theorem and the condition 3 ) of Theorem 1 for some functions $a_{0}(s)$ and $a_{1}(s)$. The assertion 2 is verified in [5] (Ch. 2, Lemma 3) for a transformation that coincides with $\tilde{A}$. The assertion 4 follows from Lemmas 14,15 , and the definition of the transformations $\tilde{B}_{n}$ if the number $n_{0}$ is sufficiently large. Therefore, for large $n_{0}$ all four assertions above are valid, and in view of Theorem 1 in $\S 2$ of [6] and Theorem 2 in $\S 1$ of $\mathrm{Ch} . \mathrm{V}$ in [7] there exist $\delta>0$ and $n_{0}$ such that if $(\varphi, z) \in \tilde{\mathcal{U}}_{\delta},|\tilde{q}-l| \leq \varkappa$, and $|\tilde{x}|<\hat{\varepsilon}$, then (85) holds, and by Lemma 14 the numbers $\delta>0$ and $n_{0}$ can be chosen to be independent of $\tilde{q}$ and $\tilde{x}$. Thus, to prove Lemma 17 it suffices to show that if $(\varphi, z) \in \tilde{\mathcal{U}}_{\delta}$, then for large $n_{0}$ the points $A^{n}\left(\varphi+\varphi_{n_{0}}, z+z_{n_{0}}\right)$ are defined. But this obviously follows from Lemmas 1,12 , and 16, the relation (85), and the condition of Lemma 17, according to which $|\tilde{x}|<\hat{\varepsilon}<a$. Lemma 17 is proved.

\section{$\S 4$. Proof of the Basic Theorem}

We consider the numbers $\varphi_{0}, m, n_{0}$ in Lemma $17(\S 3)$, and we choose the number $\varkappa>0$ in this lemma so small that $\varkappa<\hat{\varepsilon}$ and $\varkappa<\delta$, where $\hat{\varepsilon}$ and $\delta$ are also the numbers used in the statement of Lemma 17. Let $m_{0}=m+k n_{0}$, where $k$ is the integer in the statement of the Basic Theorem. Substituting the numbers $\varkappa>0, \varphi_{0}$, and $m_{0}$ into the definition of the set $\Lambda$ in the statement of the Basic Theorem, we prove that the assertions 1, 2, and 3 of the Basic Theorem hold for sufficiently small $\varkappa>0$. To this end 
we apply Lemma 17 to the transformation $A$ introduced in Definition $1(\S 3)$. Since the numbers $\varphi_{n}(n=1,2, \ldots)$ in Lemma 17 satisfy the congruence $\varphi_{n} \equiv \varphi_{0} \bmod 2 \pi$, and the right-hand sides of the system (4) are $2 \pi$-periodic in $\varphi$, we can replace $\varphi_{n_{0}}$ by $\varphi_{0}$ in Lemma 17. Furthermore, $z=\sqrt{q^{2}+p^{2}+1}$ in view of the equalities (3). Therefore, it follows from Lemma 17 that for sufficiently small $\varkappa$ the assertions 1 and 3 of the Basic Theorem are valid. We now consider the points $\left(\varphi^{(n)}, z^{(n)}\right)=A^{n}(\varphi, z)$ in Lemma 17 . The values of $x, y, q, p$ corresponding to $\varphi^{(n)}$ and $z^{(n)}$ are denoted by

$$
x^{(n)}=x\left(\varphi^{(n)}\right), \quad y^{(n)}=y\left(\varphi^{(n)}\right), \quad q^{(n)}=q\left(\varphi^{(n)}\right), \quad p^{(n)}=p\left(\varphi^{(n)}\right) .
$$

From Lemmas 1,12 , and 17 it follows that for all $n \geq 1$ the quantities $x^{(n)}$ and $y^{(n)}$ satisfy the relations

$$
\left|x^{(n)}\right| \leq a, \quad y^{(n)}=b,
$$

and, substituting the values $\tilde{z}=z^{(n)}$ and $\tilde{\varphi}=\varphi^{(n)}$ in (5), we get that for those $\varphi>\varphi^{(n)}$ such that $(x, y) \notin \Pi$ in the interval between $\varphi^{(n)}$ and $\varphi$ the values $p(\varphi)$ and $q(\varphi)$ satisfy the equalities

$$
\left\{\begin{array}{l}
p(\varphi)=\sqrt{\left(z^{(n)}\right)^{2}-1} \cos \left(\frac{\Omega\left(\varphi-\varphi^{(n)}+\hat{\varphi}_{n}\right)}{z^{(n)}}\right), \\
q(\varphi)=\sqrt{\left(z^{(n)}\right)^{2}-1} \sin \left(\frac{\Omega\left(\varphi-\varphi^{(n)}+\hat{\varphi}_{n}\right)}{z^{(n)}}\right)
\end{array}\right.
$$

where

$$
\hat{\varphi}_{n}=\frac{z^{(n)}}{\Omega} \arcsin \frac{q^{(n)}}{\sqrt{\left(z^{(n)}\right)^{2}-1}} .
$$

From the second equality in (7) and (86) it follows that the number $q^{(n)}$ does not depend on $n$, and therefore by Lemma 17 and (88)

$$
0<\frac{\Omega \hat{\varphi}_{n}}{z^{(n)}}<\frac{\pi}{2}
$$

for small $\varkappa>0$ and large $n$. Further, from the proof of Lemma 1 and the inequality (11) we get that the value $\varphi^{(n) \prime}>\varphi^{(n)}$ of $\varphi$ with $\left(x\left(\varphi^{(n) \prime}\right), y\left(\varphi^{(n) \prime}\right)\right) \in \Pi$ that is closest to $\varphi^{(n)}$ satisfies the inequality

Therefore, if

$$
\frac{3 \pi}{2}<\frac{\Omega\left(\varphi^{(n) \prime}-\varphi^{(n)}+\hat{\varphi}_{n}\right)}{z^{(n)}}<2 \pi+\frac{\pi}{2} .
$$

$$
\frac{\pi}{2} \leq \frac{\Omega\left(\varphi-\varphi^{(n)}+\hat{\varphi}_{n}\right)}{z^{(n)}} \leq \frac{3 \pi}{2},
$$

then $(x(\varphi), y(\varphi)) \notin \Pi$, and by (87) there exist values $\varphi=\varphi^{\prime}$ and $\varphi=\varphi^{\prime \prime}$ satisfying (90) such that

$$
p\left(\varphi^{\prime}\right)=-\sqrt{\left(z^{(n)}\right)^{2}-1}, \quad q\left(\varphi^{\prime \prime}\right)=\sqrt{\left(z^{(n)}\right)^{2}-1},
$$

and hence $\varlimsup_{\varphi \rightarrow \infty}|p(\varphi)|=\varlimsup_{\varphi \rightarrow \infty}|q(\varphi)|=\infty$. If we now substitute the values $\varphi=\varphi^{\prime}$, $\tilde{\varphi}=\varphi^{(n)}, \hat{\varphi}=\hat{\varphi}_{n}$, and $\tilde{z}=z^{(n)}$ in the equality (8) and the values $\varphi=\varphi^{\prime \prime}, \tilde{\varphi}=\varphi^{(n)}$, $\hat{\varphi}=\hat{\varphi}_{n}$, and $\tilde{z}=z^{(n)}$ in the equality (9), then in view of (86), (87), (88), (89), (91), and Lemma 17 we get that $\overline{\lim }_{\varphi \rightarrow \infty}|x(\varphi)|=\varlimsup_{\varphi \rightarrow \infty}|y(\varphi)|=\infty$. Thus, the assertion 2 of the Basic Theorem is proved.

We prove the assertion 4 in the case when $\tilde{y}=\tilde{y}^{*}=b$. It follows from the above proofs of the assertions 1,2 , and 3 that these assertions hold for a fixed sufficiently small $\varkappa$ and for all sufficiently large values of $m_{0}>m^{*}$. Using Lemma 17 and the fact that $\tilde{y}=\tilde{y}^{*}=b$, 
we get in this case that the points $\left(\varphi^{(n)}, z^{(n)}\right)=A^{n}(\tilde{\varphi}, \tilde{z})\left(\varphi^{(n) *}, z^{(n) *}\right)=A^{n}\left(\tilde{\varphi}, \tilde{z}^{*}\right)$ $\left(\tilde{z}=\sqrt{\tilde{q}^{2}+\tilde{p}^{2}+1}, \tilde{z}^{*}=\sqrt{\left(\tilde{q}^{*}\right)^{2}+\left(\tilde{p}^{*}\right)^{2}+1}\right)$ satisfy the inequalities

$$
\left\{\begin{array}{l}
\left|\varphi^{(n)}-\left(\varphi_{0}+2 \pi\left(m_{0}+k(n-1)\right)\right)\right| \leq \varepsilon \\
\left|\varphi^{(n) *}-\left(\varphi_{0}+2 \pi\left(m_{0}+k(n-1)\right)\right)\right| \leq \varepsilon \\
\left|z^{(n)}-\left(m_{0}+n k\right) \Omega\right| \leq \varepsilon \\
\left|z^{(n) *}-\left(m_{0}+n k\right) \Omega\right| \leq \varepsilon
\end{array}\right.
$$

and hence

$$
\left|\varphi^{(n)}-\varphi^{(n) *}\right|+\left|z^{(n)}-z^{(n) *}\right| \leq 4 \varepsilon .
$$

The values of the coordinates $y^{(n)}=y\left(\varphi^{(n)}\right), q^{(n)}=q\left(\varphi^{(n)}\right), y^{(n) *}=y\left(\varphi^{(n) *}\right), q^{(n) *}=$ $q\left(\varphi^{(n) *}\right)$ corresponding to the values $\varphi^{(n)}, z^{(n)}, \varphi^{(n) *}, z^{(n) *}$ have the form

$$
y^{(n)}=y^{(n) *}=b, \quad q^{(n)}=\tilde{q}, \quad q^{(n) *}=\tilde{q}^{*},
$$

and it follows from (92), Lemma 12 , and the equality $p^{2}(\varphi)+q^{2}(\varphi)+1=z^{2}(\varphi)$ that for a small number $\varkappa$ and a large number $m_{0}$ the coordinates

$$
x^{(n)}=x\left(\varphi^{(n)}\right), \quad p^{(n)}=p\left(\varphi^{(n)}\right), \quad x^{(n) *}=x\left(\varphi^{(n) *}\right), \quad p^{(n) *}=p\left(\varphi^{(n) *}\right)
$$

satisfy the inequalities

$$
\left\{\begin{array}{l}
\left|x^{(n)}-\tilde{x}\right| \leq \varepsilon \\
\left|x^{(n) *}-\tilde{x}^{*}\right| \leq \varepsilon \\
\left|p^{(n)}-\left(m_{0}+n k\right) \Omega\right| \leq 4 \varepsilon \\
\left|p^{(n) *}-\left(m_{0}+n k\right) \Omega\right| \leq 4 \varepsilon .
\end{array}\right.
$$

Therefore, by virtue of (95) and (7) we have

$$
\left|x^{(n)}-x^{(n) *}\right|+\left|q^{(n)}-q^{(n) *}\right|+\left|p^{(n)}-p^{(n) *}\right| \leq C^{(1)} \varepsilon,
$$

where $C^{(1)}$ is a constant that does not depend on $n$.

We consider the points

$$
\left(\varphi^{(n) \prime}, z^{(n) \prime}\right)=A^{\prime}\left(\varphi^{(n)}, z^{(n)}\right), \quad\left(\varphi^{(n) * \prime}, z^{(n) * \prime}\right)=A^{\prime}\left(\varphi^{(n) *}, z^{(n) *}\right),
$$

where $A^{\prime}$ is the mapping in Definition 1 ( $(3)$. From the definition of the transformation $A^{\prime}$ it follows that

$$
z^{(n) \prime}=z^{(n)}, \quad z^{(n) * \prime}=z^{(n) *},
$$

and we get from (16), (15), (88), and (94) that

$$
\begin{aligned}
\lim _{n \rightarrow \infty}\left[\varphi^{(n) \prime}-\left(\varphi^{(n)}+\frac{2 \pi z^{(n)}}{\Omega}-2 \hat{\varphi}_{n}\right)\right] & =-2 b+\frac{2 \tilde{q}}{\Omega}, \\
\lim _{n \rightarrow \infty}\left[\varphi^{(n) * \prime}-\left(\varphi^{(n) *}+\frac{2 \pi z^{(n) *}}{\Omega}-2 \hat{\varphi}_{n}^{*}\right)\right] & =-2 b+\frac{2 \tilde{q}^{*}}{\Omega},
\end{aligned}
$$

where

$$
\hat{\varphi}_{n}^{*}=\frac{z^{(n) *}}{\Omega} \arcsin \frac{q^{(n) *}}{\sqrt{\left(z^{(n) *}\right)^{2}-1}} .
$$

Therefore, subtracting the equality (98) from the equality (99), we get from (93), (96), (88), and (100) that for small $\varkappa$ and large $m_{0}$

$$
\left|\varphi^{(n) \prime}-\varphi^{(n) * \prime}\right|<C^{(2)} \varepsilon,
$$

where $C^{(2)}$ is a constant that does not depend on $n$. 
We prove the assertion 4 for those values of $\varphi$ with

$$
\max \left\{\varphi^{(n)}, \varphi^{(n) *}\right\}<\varphi<\min \left\{\varphi^{(n) \prime}, \varphi^{(n) * \prime}\right\} .
$$

If the inequalities (102) hold, then $(x(\varphi), y(\varphi)) \notin \Pi$ and $\left(x^{*}(\varphi), y^{*}(\varphi)\right) \notin \Pi$ for both solutions, and thus the functions $p(\varphi)$ and $q(\varphi)$ for the first solution corresponding to the point $\left(\varphi^{(n)}, z^{(n)}\right)$ have the form (87), and the functions $p^{*}(\varphi)$ and $q^{*}(\varphi)$ for the second solution corresponding to the point $\left(\varphi^{(n) *}, z^{(n) *}\right)$ have the form

$$
\left\{\begin{array}{l}
p^{*}(\varphi)=\sqrt{\left(z^{(n) *}\right)^{2}-1} \cos \left(\frac{\Omega\left(\varphi-\varphi^{(n) *}+\hat{\varphi}_{n}^{*}\right)}{z^{(n) *}}\right), \\
q^{*}(\varphi)=\sqrt{\left(z^{(n) *}\right)^{2}-1} \sin \left(\frac{\Omega\left(\varphi-\varphi^{(n) *}+\hat{\varphi}_{n}^{*}\right)}{z^{(n) *}}\right)
\end{array}\right.
$$

where $\hat{\varphi}_{n}^{*}$ is the number defined in (100).

Since the derivatives of the functions $p(\varphi)$ and $q(\varphi)$ in (87) with respect to the variables $\varphi, z^{(n)}$, and $\varphi^{(n)}$ are bounded in modulus by a constant independent of $n$, it follows in view of $(87),(103),(93),(88),(100)$, and (96) that for small $\varkappa$ and large $m_{0}$ the inequality

$$
\begin{aligned}
\left|p\left(\varphi^{(n) \prime}\right)-p^{*}\left(\varphi^{(n) * \prime}\right)\right| & +\left|q\left(\varphi^{(n) \prime}\right)-q^{*}\left(\varphi^{(n) * \prime}\right)\right| \\
& +\left|p(\varphi)-p^{*}(\varphi)\right|+\left|q(\varphi)-q^{*}(\varphi)\right|<C^{(3)} \varepsilon
\end{aligned}
$$

holds in the domain (102), where $C^{(3)}$ is a constant independent of $n$.

If we now use the equalities (7) and the inequalities (96) and (104) in the domain (102), then we get that

$$
\begin{aligned}
\left|x\left(\varphi^{(n) \prime}\right)-x^{*}\left(\varphi^{(n) * \prime}\right)\right| & +\left|y\left(\varphi^{(n) \prime}\right)-y^{*}\left(\varphi^{(n) * \prime}\right)\right| \\
& +\left|x(\varphi)-x^{*}(\varphi)\right|+\left|y(\varphi)-y^{*}(\varphi)\right|<C^{(4)} \varepsilon
\end{aligned}
$$

where $C^{(4)}$ is a constant independent of $n$.

The inequalities (104) and (105) prove the assertion 4 of the Basic Theorem in the domain (102) for all sufficiently large values of $n$ under the condition that $\tilde{y}=\tilde{y}^{*}=b$. We prove that here the conditions of the assertion 4 are valid in the domains

$$
\min \left\{\varphi^{(n)}, \varphi^{(n) *}\right\} \leq \varphi \leq \max \left\{\varphi^{(n)}, \varphi^{(n) *}\right\}
$$

and

$$
\min \left\{\varphi^{(n) \prime}, \varphi^{(n) * \prime}\right\} \leq \varphi<\min \left\{\varphi^{(n+1)}, \varphi^{(n+1) *}\right\} .
$$

Suppose that $\varphi$ satisfies (106) and assume for definiteness that

$$
\min \left\{\varphi^{(n)}, \varphi^{(n) *}\right\}=\varphi^{(n)} .
$$

We have

$$
\begin{aligned}
\left|x(\varphi)-x^{*}(\varphi)\right| & +\left|y(\varphi)-y^{*}(\varphi)\right|+\left|p(\varphi)-p^{*}(\varphi)\right| \\
& +\left|q(\varphi)-q^{*}(\varphi)\right| \leq \Sigma^{(1)}+\Sigma^{(2)}+\Sigma^{(3)}
\end{aligned}
$$

where

$$
\begin{aligned}
& \Sigma^{(1)}=\left|x(\varphi)-x\left(\varphi^{(n)}\right)\right|+\left|y(\varphi)-y\left(\varphi^{(n)}\right)\right|+\left|p(\varphi)-p\left(\varphi^{(n)}\right)\right|+\left|q(\varphi)-q\left(\varphi^{(n)}\right)\right| \\
& \Sigma^{(2)}=\left|x\left(\varphi^{(n)}\right)-x^{*}\left(\varphi^{(n) *}\right)\right|+\left|y\left(\varphi^{(n)}\right)-y^{*}\left(\varphi^{(n) *}\right)\right| \\
&+\left|p\left(\varphi^{(n)}\right)-p^{*}\left(\varphi^{(n) *}\right)\right|+\left|q\left(\varphi^{(n)}\right)-q^{*}\left(\varphi^{(n) *}\right)\right| \\
& \Sigma^{(3)}=\left|x^{*}(\varphi)-x^{*}\left(\varphi^{(n) *}\right)\right|+\left|y^{*}(\varphi)-y^{*}\left(\varphi^{(n) *}\right)\right| \\
&+\left|p^{*}(\varphi)-p^{*}\left(\varphi^{(n) *}\right)\right|+\left|q^{*}(\varphi)-q^{*}\left(\varphi^{(n) *}\right)\right|
\end{aligned}
$$


Let us estimate the quantities $\Sigma^{(i)}(i=1,2,3)$. Since the quantities $x\left(\varphi^{(n)}\right), y\left(\varphi^{(n)}\right)$, $p\left(\varphi^{(n)}\right), q\left(\varphi^{(n)}\right)$ are equal to the respective quantities $x^{(n)}, b, p^{(n)}, q^{(n)}$, and the quantities $x^{*}\left(\varphi^{(n) *}\right), y^{*}\left(\varphi^{(n) *}\right), p^{*}\left(\varphi^{(n) *}\right), q^{*}\left(\varphi^{(n) *}\right)$ are equal to the respective quantities $x^{*(n)}, b$, $p^{*(n)}, q^{*(n)}$, it follows from the inequalities (96) and (111) that $\Sigma^{(2)} \leq C^{(1)} \varepsilon$. By (108), for small $\varepsilon$ we have $(x(\varphi), y(\varphi)) \notin \Pi$ for $\varphi^{(n)}<\varphi \leq \varphi^{(n) *}$, and $\left(x^{*}(\varphi), y^{*}(\varphi)\right) \in \Pi$ for $\varphi^{(n)} \leq \varphi \leq \varphi^{(n) *}$. Moreover, it follows from (106) and (93) that $\left|\varphi-\varphi^{(n)}\right|+\left|\varphi-\varphi^{*(n)}\right| \leq$ $4 \varepsilon$. Therefore, since in (106) the derivatives of the functions $x(\varphi), y(\varphi), p(\varphi), q(\varphi), x^{*}(\varphi)$, $y^{*}(\varphi), p^{*}(\varphi), q^{*}(\varphi)$ are bounded in modulus by a constant independent of $n$, we get from (110) and (112) the estimate

$$
\Sigma^{(1)}+\Sigma^{(3)}<C^{(5)} \varepsilon,
$$

where $C^{(5)}$ is a constant independent of $n$. Substitution of these estimates for the quantities $\Sigma^{(i)}(i=1,2,3)$ in (109) proves the assertion 4 in the domain (106).

Assume now that $\varphi$ satisfies (107), and for definiteness that $\min \left\{\varphi^{(n) \prime}, \varphi^{(n) * \prime}\right\}=$ $\varphi^{(n) \prime}$. In this case for small $\varepsilon$ we have $(x(\varphi), y(\varphi)) \in \Pi$ for $\varphi^{(n) \prime} \leq \varphi \leq \varphi^{(n) * \prime}$, and $\left(x^{*}(\varphi), y^{*}(\varphi)\right) \notin \Pi$ for $\varphi^{(n) \prime} \leq \varphi<\varphi^{(n) * \prime}$. Moreover, the inequality (109) holds, in which the quantities $\Sigma^{(1)}, \Sigma^{(2)}$, and $\Sigma^{(3)}$ are obtained from the corresponding quantities in (110)-(112) by replacing $\varphi^{(n)}$ by $\varphi^{(n) \prime}$ and $\varphi^{(n) *}$ by $\varphi^{(n) * \prime}$.

By (104) and (105), the quantity $\Sigma^{(2)}$ obtained in this way admits the estimate

$$
\Sigma^{(2)} \leq\left(C^{(3)}+C^{(4)}\right) \varepsilon,
$$

and from (97) and the inequality

$$
\left|\varphi-\varphi^{(n) \prime}\right|+\left|\varphi-\varphi^{(n) * \prime}\right|<C^{(2)} \varepsilon
$$

(which is a consequence of the inequalities (101) and (107)) it follows in view of the boundedness in modulus of the derivatives of the functions $x(\varphi), y(\varphi), p(\varphi), q(\varphi), x^{*}(\varphi)$, $y^{*}(\varphi), p^{*}(\varphi), q^{*}(\varphi)$ by a constant independent of $n$ that

$$
\Sigma^{(1)}+\Sigma^{(3)}<C^{(6)} \varepsilon,
$$

where $C^{(6)}$ is a constant independent of $n$. Substitution of these estimates for $\Sigma^{(i)}(i=$ $1,2,3)$ in (109) proves the assertion 4 in the domain (107) for the case $\tilde{y}=\tilde{y}^{*}=b$.

Assume now that, as indicated in the formulation of the Basic Theorem, $\tilde{y}$ and $\tilde{y}^{*}$ satisfy the inequalities $|\tilde{y}-b| \leq \varkappa$ and $\left|\tilde{y}^{*}-b\right| \leq \varkappa$. Then for a small value of the constant $\varkappa>0$ we consider instead of $\tilde{\varphi}$ the value $\bar{\varphi}$ of $\varphi$ such that if $\tilde{y}<b$ and $\overline{\bar{\varphi}}$ is the value of $\varphi$ closest to $\tilde{\varphi}$ that satisfies $\overline{\bar{\varphi}}>\tilde{\varphi}$ and $y(\bar{\varphi})=b$, then $\bar{\varphi}$ is the value of $\varphi$ closest to $\overline{\bar{\varphi}}$ such that $\bar{\varphi}>\overline{\bar{\varphi}}$ and the solution with initial data $\tilde{\varphi}, \tilde{x}, \tilde{y}, \tilde{q}, \tilde{p}$ satisfies the equality $y(\bar{\varphi})=b$, while if $\tilde{y}>b$, then $\bar{\varphi}$ is the value of $\varphi$ closest to $\tilde{\varphi}$ such that $\bar{\varphi}>\tilde{\varphi}$ and this solution satisfies the equality $y(\bar{\varphi})=b$. In exactly the same way we introduce the value $\bar{\varphi}^{*}$ for the solution with initial data $\tilde{\varphi}, \tilde{x}^{*}, \tilde{y}^{*}, \tilde{q}^{*}, \tilde{p}^{*}$. If we now denote by $\bar{z}$ and $\bar{z}^{*}$ the values $z(\bar{\varphi})$ and $z\left(\bar{\varphi}^{*}\right)$ of the variable $z$ corresponding to $\bar{\varphi}$ and $\bar{\varphi}^{*}$ and substitute the points $(\bar{\varphi}, \bar{z})$ and $\left(\bar{\varphi}^{*}, \bar{z}^{*}\right)$ in place of the points $(\tilde{\varphi}, \tilde{z})$ and $\left(\tilde{\varphi}, \tilde{z}^{*}\right)$ in the proof of the assertion 4 for the case $\tilde{y}=\tilde{y}^{*}=b$, then for small $\varkappa$ and large $m_{0}$ we get the assertion 4 for the case when $|\tilde{y}-b| \leq \varkappa$ and $\left|\tilde{y}^{*}-b\right| \leq \varkappa$. The Basic Theorem is proved.

\section{§5. Hamiltonian Systems With SMOOTH RIGHT-HAND SIDES}

The right-hand sides of the systems (I) and (IV) are discontinuous with respect to $x$ and $y$, because the functions $G(y, \varphi)$ and $D(x, y, \varphi)$ in (III) and (V) are discontinuous with respect $x$ and $y$.

In this section we indicate infinitely differentiable approximations of the system (I) for which the assertions of Theorem 1 are valid. 
For an infinite sequence $\varkappa_{n}(n=1,2, \ldots)$ with $\varkappa_{n}>0$ and $\lim _{n \rightarrow \infty} \varkappa_{n}=0$ we take the sequence of rectangles $\Pi_{n}$ given by

$$
\Pi_{n}=\left\{x, y:|x| \leq a+\varkappa_{n},|y| \leq b+\varkappa_{n}\right\} .
$$

For any $n=1,2, \ldots$ we consider an infinitely differentiable function $g_{n}=g_{n}(x, y, \varphi)$ with the following properties:

a) $g_{n}(x, y, \varphi)$ has period $2 \pi$ with respect to $\varphi$;

b)

$$
g_{n}(x, y, \varphi)=\left\{\begin{array}{lll}
G(y, \varphi) & \text { if } \quad(x, y) \in \Pi, \\
\tilde{g}(y, \varphi) & \text { if } \quad|x| \leq a, \quad b \leq|y| \leq b+\varkappa_{n}, \\
0 & \text { if } \quad(x, y) \notin \Pi_{n},
\end{array}\right.
$$

where $G$ is the function in (III);

c)

$$
\left|g_{n}(x, y, \varphi)\right|<C^{*}
$$

where $C^{*}$ is a constant independent of $x, y, \varphi$, and $n$.

We introduce a sequence of infinitely differentiable functions $f_{n}=f_{n}(x, y, \varphi)(n=$ $1,2, \ldots)$ such that if $|x| \leq a$ and $|y| \leq b+\varkappa_{n}$, then

$$
\frac{\partial f_{n}}{\partial y}(x, y, \varphi)=g_{n}(x, y, \varphi), \quad \frac{\partial f_{n}}{\partial x}(x, y, \varphi) \equiv 0,
$$

if $(x, y) \in \Pi$, then $f_{n}(x, y)$ does not depend on $n$, and if $(x, y) \notin \Pi_{n}$, then

$$
\frac{\partial f_{n}}{\partial y}(x, y, \varphi)=\frac{\partial f_{n}}{\partial x}(x, y, \varphi)=0 .
$$

Further, if the number $\tilde{\varkappa}>0$ is sufficiently small and $\tilde{m}$ is a fixed integer, then there exist sequences of infinitely differentiable functions $G_{n}=G_{n}(x, y, Q, P, \varphi)$ and $F_{n}=$ $F_{n}(x, y, Q, P, \varphi)(n=1,2, \ldots)$ defined in five-dimensional space such that if $|x| \leq a$, $b \leq|y| \leq b+\varkappa_{n}$, and

$$
\left|\sqrt{1+\left(Q+\frac{\Omega y}{2}\right)^{2}+\left(P-\frac{\Omega x}{2}\right)^{2}}-(\tilde{m}+n k) \Omega\right| \leq \tilde{\varkappa},
$$

then

$$
\begin{aligned}
& G_{n}(x, y, Q, P, \varphi)=g_{n}(x, y, \varphi), \\
& F_{n}(x, y, Q, P, \varphi)=f_{n}(x, y, \varphi),
\end{aligned}
$$

if $(x, y) \in \Pi$, then (116) and (117) again hold, and if $(x, y) \notin \Pi_{n}$ and (115) holds, then

$$
\begin{aligned}
G_{n}(x, y, Q, P, \varphi) & =\frac{\partial F_{n}}{\partial x}(x, y, Q, P, \varphi)=\frac{\partial F_{n}}{\partial y}(x, y, Q, P, \varphi) \\
& =\frac{\partial F_{n}}{\partial Q}(x, y, Q, P, \varphi)=\frac{\partial F_{n}}{\partial P}(x, y, Q, P, \varphi)=0 .
\end{aligned}
$$

From the construction of the functions $g_{n}, f_{n}, G_{n}$, and $F_{n}$ it follows that if $\tilde{\varkappa}$ is sufficiently small, then there exist infinitely differentiable functions

$$
G^{*}=G^{*}(x, y, Q, P, \varphi), \quad F^{*}=F^{*}(x, y, Q, P, \varphi)
$$


having period $2 \pi$ with respect to $\varphi$ such that for any $n=1,2, \ldots$ if $|x| \leq a, b \leq|y| \leq$ $b+\varkappa_{n}$, and (115) holds, then

$$
\begin{aligned}
& G^{*}(x, y, Q, P, \varphi)=G_{n}(x, y, Q, P, \varphi), \\
& F^{*}(x, y, Q, P, \varphi)=F_{n}(x, y, Q, P, \varphi),
\end{aligned}
$$

if $(x, y) \in \Pi$, then (118) and (119) again hold, and if $(x, y) \notin \Pi_{n}$ and (115) holds, then the value of $G^{*}$ and the values of the derivatives of $F^{*}$ with respect to $x, y, Q$, and $P$ are equal to zero.

It follows from the definitions of the functions $G^{*}$ and $F^{*}$ that if $(x, y) \in \Pi$, then

$$
\frac{\partial F^{*}}{\partial y}(x, y, Q, P, \varphi)=G^{*}(x, y, Q, P, \varphi)=G(y, \varphi),
$$

where $G(y, \varphi)$ is the function in (III), which is analytic with respect to $y$ and $\varphi$ and has period $2 \pi$ with respect to $\varphi$.

We consider the Hamiltonian system (I) with Hamiltonian function

$$
H=\sqrt{1+\left(Q+\frac{\Omega y}{2}\right)^{2}+\left(P-\frac{\Omega x}{2}\right)^{2}}+F^{*}(x, y, Q, P, \varphi),
$$

where $F^{*}(x, y, Q, P, \varphi)$ is the infinitely differentiable function constructed above.

The construction of the functions $G^{*}$ and $F^{*}$ and the Hamiltonian function $H$ in (120) is realized if we specify a sequence of positive numbers $\varkappa_{n}(n=1,2, \ldots)$, a function $G(y, \varphi)$ in (113), a sufficiently small number $\tilde{\varkappa}>0$, and an integer $\tilde{m}$ in (115).

Theorem 2. Suppose that the function $G(y, \varphi)$ in (113) satisfies all the conditions of Theorem $1(\S 1)$. Then there is a sequence of numbers $\varkappa_{n}>0(n=1,2, \ldots)$ such that all the assertions of Theorem 1 are valid for the system (I) with the infinitely differentiable Hamiltonian function $H$ in (120).

Remark. It follows from the proof of Theorem 2 that it is valid for any sequence of positive numbers $\varkappa_{n}$ that decreases sufficiently rapidly as $n \rightarrow \infty$.

Proof of Theorem 2. Let the variables $q$ and $p$ be defined by (IV) $(\S 1)$. In the variables $x, y, q, p, \varphi$ the system of equations (I) with the Hamiltonian function (120) takes the form of the system of equations

$$
\begin{cases}\frac{d x}{d \varphi}=\frac{q}{\sqrt{1+p^{2}+q^{2}}}+\Psi^{(1)}, & \frac{d y}{d \varphi}=\frac{p}{\sqrt{1+p^{2}+q^{2}}}+\Psi^{(2)} \\ \frac{d q}{d \varphi}=\frac{\Omega p}{\sqrt{1+p^{2}+q^{2}}}+\Psi^{(3)}, & \frac{d p}{d \varphi}=-\frac{\Omega q}{\sqrt{1+p^{2}+q^{2}}}+\Psi^{(4)}\end{cases}
$$

moreover, the equalities $\Psi^{(s)}=0(s=1,2,3,4)$ hold in the domains $(x, y) \notin \Pi_{n}$, $\left|\sqrt{1+p^{2}+q^{2}}-(\tilde{m}+n k) \Omega\right| \leq \tilde{\varkappa}$, the equalities $\Psi^{(1)}=\Psi^{(2)}=\Psi^{(3)}=0$ and $\Psi^{(4)}=$ $E(y, \varphi)=-G(y, \varphi)$ hold in the domain $(x, y) \in \Pi$, where $G(y, \varphi)$ is the function in (113), and the functions $\Psi^{(s)}(s=1,2,3,4)$ satisfy the inequalities

$$
\left|\Psi^{(s)}\right|<C^{*}
$$

in the domains $|x| \leq a, b \leq|y| \leq b+\varkappa_{n}$, where $C^{*}$ is the constant in (114).

It follows from the formulation of Theorem 2 that it suffices to prove all the assertions of the Basic Theorem $(\S 2)$ in connection with the system (121). The proof given in $\S 4$ for the Basic Theorem in the case of the system (4) was based on Lemma 17 (§3). But from the definitions of the functions $G^{*}$ and $F^{*}$ and from the form of the system of equations (121) it follows in view of (122) that if the sequence of positive numbers $\varkappa_{n}$ tends to zero 
sufficiently rapidly, then the assertion of Lemma 17 is also valid in connection with the system (121), and this proves Theorem 2.

\section{$\S 6$. Preservation of Stability of solutions under a small Change OF THE HAMILTONIAN}

We introduce a norm in the space of smooth functions of the five variables $x, y, Q, P$, $\varphi$. To this end we specify positive numbers $a, b, \delta, \varphi_{0}, \Omega$ and a positive integer $k$, and for any positive integer $n$ we define domains $\Gamma_{n}$ and $K_{n}$ as follows:

$$
\begin{gathered}
\Gamma_{n}=\left\{x, y, Q, P, \varphi:\left|\sqrt{1+\left(Q+\frac{\Omega y}{2}\right)^{2}+\left(P-\frac{\Omega x}{2}\right)^{2}}-n k \Omega\right| \leq \delta\right\}, \\
K_{n}=\left\{x, y, Q, P, \varphi:|x| \leq a,|y| \leq b,-2 \Omega b-\delta+\varphi_{0} \Omega \leq q+\frac{\Omega y}{2} \leq \delta+\varphi_{0} \Omega,\right. \\
\left.n k \Omega-\delta \leq \sqrt{1+\left(Q+\frac{\Omega y}{2}\right)^{2}+\left(P-\frac{\Omega x}{2}\right)^{2}} \leq(n+1) k \Omega+\delta\right\} .
\end{gathered}
$$

Definition 5. For any given smooth function $W=W(x, y, Q, P, \varphi)$ we define a norm $\|W\|$ as follows:

$$
\|W\|=\sum_{n=1}^{\infty}\left(\sup _{(x, y, Q, P, \varphi) \in K_{n}}|\nabla W|+n \sup _{(x, y, Q, P, \varphi) \in \Gamma_{n}}|\nabla W|\right),
$$

where $\nabla W=\left(\frac{\partial W}{\partial x}, \frac{\partial W}{\partial y}, \frac{\partial W}{\partial Q}, \frac{\partial W}{\partial P}\right)$ is the gradient vector of the function $W$, and $|\nabla W|$ is its length.

Theorem 3. Consider the Hamiltonian system (1) with Hamiltonian function

$$
\begin{aligned}
H= & H(x, y, Q, P, \varphi) \\
= & \sqrt{1+\left(Q+\frac{\Omega y}{2}\right)^{2}+\left(P-\frac{\Omega x}{2}\right)^{2}} \\
& +F(x, y, \varphi)+W(x, y, Q, P, \varphi),
\end{aligned}
$$

where $W(x, y, Q, P, \varphi)$ is an infinitely differentiable function, and $F(x, y, \varphi)$ is the function in (II), which satisfies the conditions a), b), and c) (§1).

In this case if $\|W\|<\infty$, then for the solutions of the system (I) with Hamiltonian function (123) the assertions 1-3 of Theorem 1 are valid, and for any $\varepsilon>0$ there exist numbers $\varkappa>0$ and $m^{*}>0$ such that if $\|W\|<\varkappa, m_{0}>m^{*}, \Gamma\left(\varkappa, m_{0}\right)$ is the domain in the statement of Theorem $1,(\tilde{x}, \tilde{y}, \tilde{Q}, \tilde{P}, \tilde{\varphi}) \in \Gamma\left(\varkappa, m_{0}\right)$, and $\left(\tilde{x}^{*}, \tilde{y}^{*}, \tilde{Q}^{*}, \tilde{P}^{*}, \tilde{\varphi}\right) \in$ $\Gamma\left(\varkappa, m_{0}\right)$, then the assertion 4 of Theorem 1 holds.

Theorem 4. The assertions of Theorem 3 are valid for the system (1) with Hamiltonian function $H$ obtained from the function defined in (123) by replacing in it the function $F(x, y, \varphi)$ by the infinitely differentiable function $F^{*}(x, y, Q, P, \varphi)$ in $\S 5$.

The proofs of Theorems 3 and 4 with the help of the change of variables (IV) reduce to proofs of the assertions of the Basic Theorem for the system of equations in the variables $x, y, q, p, \varphi$ obtained from the systems in the statements of Theorems 3 and 4 as a result of this change. The proofs of the assertions of the Basic Theorem for these systems of equations in the variables $x, y, q, p, \varphi$ reduce in turn to a proof of Lemma 17 (§3) in connection with these systems, but for this situation the lemma is valid in view of the definition of the norm $\|W\|$ in this section. 


\section{$\S 7$. THE LIMITING INTEGRABLE SYSTEM}

We consider the solution of the system (4) (§2) with initial data $\tilde{x}=x(\tilde{\varphi}), \tilde{y}=b$, $\tilde{q}=q(\tilde{\varphi}), \tilde{p}=p(\tilde{\varphi})$. Suppose that the conditions of Lemma 1 hold $(\S 3)$. Then by virtue of this lemma the transformations

$$
A:(\tilde{\varphi}, \tilde{z}) \longmapsto\left(\tilde{\varphi}^{\prime}, \tilde{z}^{\prime}\right), \quad A^{\prime}:(\tilde{\varphi}, \tilde{z}) \longmapsto\left(\varphi^{\prime}, z^{\prime}\right)
$$

are defined. In Lemma 1 it was proved that for $\tilde{\varphi}<\varphi<\varphi^{\prime}$ the condition $(x(\varphi), y(\varphi)) \notin \Pi$ holds for the solution $(x(\varphi), y(\varphi), q(\varphi), p(\varphi))$ of the system (4), and therefore in this domain the functions $q(\varphi)$ and $p(\varphi)$ have the form (5), and the functions $x(\varphi)$ and $y(\varphi)$ are given by (8) and (9). It follows from the equalities (5) that the quantity $z(\varphi)=\sqrt{q^{2}(\varphi)+p^{2}(\varphi)+1}=z(\tilde{\varphi})=\tilde{z}$ does not depend on $\varphi$.

We introduce the small parameter $\mu=\tilde{z}^{-1}=\left(\tilde{q}^{2}+\tilde{p}^{2}+1\right)^{-1 / 2}$. The parameter $\mu$ is connected with the parameter $\nu=\frac{1}{p^{\prime}}=\frac{1}{p\left(\varphi^{\prime}\right)}$ introduced in the proof of Lemma 1 and appearing in the equations (23) and (24) by the equality

$$
\mu=\frac{\nu}{\sqrt{1+\left(q^{\prime}\right)^{2}+\nu^{2}}}
$$

where $q^{\prime}=q^{\prime}\left(\varphi^{\prime}\right)$. But it follows from (17) that $\lim _{\tilde{z} \rightarrow \infty} q^{\prime}=\lim _{\tilde{p} \rightarrow \infty} q^{\prime}=-2 b \Omega+2 \tilde{q}$. Therefore, for $\varphi^{\prime} \leq \varphi \leq \tilde{\varphi}^{\prime}$ the system of equations obtained from the system (4) as $\mu \rightarrow 0$ coincides with the system obtained from the system (23), (24) when we pass to the limit as $\nu \rightarrow 0$, in view of (124). As a result of this passage to the limit we get the integrable system of equations

$$
\frac{d x}{d \varphi}=0, \quad \frac{d y}{d \varphi}=1, \quad \frac{d q}{d \varphi}=\Omega, \quad \frac{d p}{d \varphi}=E(y, \varphi)
$$

whose solution is

$$
\left\{\begin{array}{l}
x(\varphi)=x\left(\varphi^{\prime}\right), \quad y(\varphi)=y\left(\varphi^{\prime}\right)+\varphi, \quad q(\varphi)=q\left(\varphi^{\prime}\right)+\Omega \varphi, \\
p(\varphi)=p\left(\varphi^{\prime}\right)+\int_{\varphi^{\prime}}^{\varphi} E\left(y\left(\varphi^{\prime}\right)+\varphi, \varphi\right) d \varphi .
\end{array}\right.
$$

Thus, as $\mu \rightarrow 0$ the solution of the limit system is given by (5), (8), (9) for $\tilde{\varphi} \leq \varphi \leq \varphi^{\prime}$, and by (125) for $\varphi^{\prime} \leq \varphi \leq \tilde{\varphi}^{\prime}$. According to the definition of the transformation $A$ (Definition $1, \S 3$ ), we have $y\left(\tilde{\varphi}^{\prime}\right)=b$; therefore, repeating these arguments for $\varphi>\tilde{\varphi}^{\prime}$, we obtain for the system (4) the solution of the limit system as $\mu \rightarrow 0$ for all values of $\varphi \geq \tilde{\varphi}$.

Since the system of equations (I) with Hamiltonian function (II) reduces to the system (4) as a result of the change of variables (IV), it follows that for the system (1) the small parameter $\mu$ has the form

$$
\mu=\left[1+\left(Q+\frac{\Omega y}{2}\right)^{2}+\left(P-\frac{\Omega x}{2}\right)^{2}\right]^{-1 / 2} .
$$

\section{REFERENCES}

[1] V.I. Veksler, A new method for accelerating relativistic particles, Dokl. Akad. Nauk SSSR 43 (1944), 346-348. (Russian)

[2] S.P. Kapitsa and V.N. Melekhin, The microtron, "Nauka", Moscow, 1969. (Russian)

[3] L. A. Artsimovich and S. Yu. Luk'yanov, The motion of charged particles in electric and magnetic fields, 2nd ed., "Nauka", Moscow, 1978. (Russian)

[4] L. D. Pustyl'nikov, On oscillatory motions in a certain dynamical system, Izv. Akad. Nauk SSSR Ser. Mat. 51 (1987), 1010-1032; English transl, Math. USSR Izv. 31 (1988), 325-347. MR0925092 (89a:78003) 
[5] L.D. Pustyl'nikov, Stable and oscillating motions in nonatomic dynamical systems II, Trudy Moskov. Mat. Obshch. 34 (1977), 3-103; English transl. in Trans. Moscow Math. Soc. 1978, no. 2, 1-101. MF 0477274 (57:16815)

[6] L.D. Pustyl'nikov, Unbounded growth of the action variable in certain physical models, Trudy Moskov. Mat. Obshch. 46 (1983), 187-200; English transl. in Trans. Moscow Math. Soc. 1984, no. 2, 193-205. MR0737907 (85b:70019)

[7] L.D. Pustyl'nikov, Poincaré models, a rigorous proof of the second element of thermodynamics on the basis of mechanics, and the Fermi acceleration mechanism, Uspekhi Mat. Nauk 50 (1995), no. 1, 143-186; English transl. in Russian Math. Surveys 50 (1995), no. 1, 145-190. MR1331358 (96c:82035)

[8] L.D. Pustyl'nikov, On certain final motions in the n-body problem, Prikl. Mat. Mekh. 1990, no. 2, 329-331; English transl. in J. Appl. Math. Mech. 54 (1991), 272-274. MR,1065775 (91m:70012)

[9] L. D. Pustyl'nikov, On the measure of one-way oscillating motions for the Kolmogorov model and its generalization in the n-body problem, Uspekhi Mat. Nauk SSSR 53 (1998), no. 5, 245-246; English transl. in Russian Math. Surveys 53 (1998), 1102-1103. MR.1691200(2001i:70015)

[10] L. D. Pustyl'nikov, On the stability of solutions and absence of Arnol'd diffusion in a nonintegrable Hamiltonian system of a general form with three degrees of freedom, preprint no. 155, WeierstrassInstitut für Angewandte Analysis und Stochastik, Berlin, 1995.

[11] J. Chazy, Sur l'allure finale du mouvement dans le problème des trois corps quand le temps croit indéfiniment, Annales de l'École Norm. Sup. (3) 39 (1922), 29-130.

[12] K. A. Sitnikov, Existence of oscillating motions in the three-body problem, Dokl. Akad. Nauk SSSR 133 (1960), 303-306; English transl. in Soviet Phys. Dokl. 5 (1961), 647-650. MF0127389 (23:B435)

[13] V. M. Alekseev, Quasi-random dynamical systems. I, II, III, Mat. Sb. 76 (1968), 72-134; 77 (1968), 545-600; 78 (1969), 3-50; English transl. in Math. USSR Sb. 5 (1968), 73-128; 6 (1968), 505-560; 7 (1969), 1-43. MR0276948(43:2687b) MR0276949 (43:2688) MR0276950(43:2689)

[14] A. M. Leontovich, On the existence of unbounded oscillating trajectories in a billiards problem, Dokl. Akad. Nauk SSSR 145 (1962), 523-526; English transl. in Soviet Math. Dokl. 3 (1962), 1049-1052. MR $0138846(25: 2287)$

[15] V.I. Arnol'd, On the instability of dynamical systems with several degrees of freedom, Dokl. Akad. Nauk SSSR 156 (1964), 9-12; English transl. in Soviet Math. Dokl. 5 (1964), 581-585. MR0163026 $(29: 329)$

[16] C.L. Siegel, Vorlesungen über Himmelsmechanik, Springer-Verlag, Berlin, 1956; Russian transl., Inostran. Lit., Moscow, 1959; rev. aug. English transl. (with J. K. Moser), Springer-Verlag, Berlin, 1971. MR.0080009 (18:178c)

Keldysh Institute of Applied Mathematics, Russian Academy of Sciences, Miusskaya Pl. 4, Moscow 125047, Russia

E-mail address: lpustyln@spp.keldysh.ru 